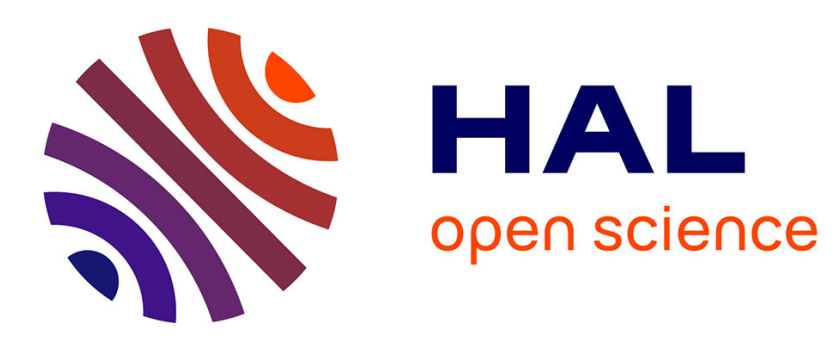

\title{
Time-domain simulation of damped impacted plates. II. Numerical model and results
}

\author{
Christophe Lambourg, Antoine Chaigne, Denis Matignon
}

\section{To cite this version:}

Christophe Lambourg, Antoine Chaigne, Denis Matignon. Time-domain simulation of damped impacted plates. II. Numerical model and results. Journal of the Acoustical Society of America, 2001, vol. 109 (n 4), pp. 1433-1447. 10.1121/1.1354201 . hal-01374311

\section{HAL Id: hal-01374311 \\ https://hal.science/hal-01374311}

Submitted on 30 Sep 2016

HAL is a multi-disciplinary open access archive for the deposit and dissemination of scientific research documents, whether they are published or not. The documents may come from teaching and research institutions in France or abroad, or from public or private research centers.
L'archive ouverte pluridisciplinaire HAL, est destinée au dépôt et à la diffusion de documents scientifiques de niveau recherche, publiés ou non, émanant des établissements d'enseignement et de recherche français ou étrangers, des laboratoires publics ou privés. 


\title{
Time-domain simulation of damped impacted plates. II. Numerical model and results
}

\author{
Christophe Lambourg, ${ }^{\text {a) }}$ Antoine Chaigne, ${ }^{\text {b) }}$ and Denis Matignon \\ Ecole Nationale Supérieure des Télécommunications, Département TSI, CNRS URA 820, 46 Rue Barrault, \\ 75634 Paris Cedex 13, France
}

\begin{abstract}
A time-domain model for the flexural vibrations of damped plates was presented in a companion paper [Part I, J. Acoust. Soc. Am. 109, 1422-1432 (2001)]. In this paper (Part II), the damped-plate model is extended to impact excitation, using Hertz's law of contact, and is solved numerically in order to synthesize sounds. The numerical method is based on the use of a finite-difference scheme of second order in time and fourth order in space. As a consequence of the damping terms, the stability and dispersion properties of this scheme are modified, compared to the undamped case. The numerical model is used for the time-domain simulation of vibrations and sounds produced by impact on isotropic and orthotropic plates made of various materials (aluminum, glass, carbon fiber and wood). The efficiency of the method is validated by comparisons with analytical and experimental data. The sounds produced show a high degree of similarity with real sounds and allow a clear recognition of each constitutive material of the plate without ambiguity. (C) 2001 Acoustical Society of America.
\end{abstract}

PACS numbers: 43.40.Dx [CBB]

\section{INTRODUCTION}

A time-domain model of damped isotropic and orthotropic plates was derived in a companion paper (Part I) in order to investigate the relevance of material properties in the quality of sounds produced by vibrating structures. This plate model includes damping terms expressed by means of a general differential operator similar to the one commonly used in viscoelasticity. In the previous paper it was shown under which conditions this operator is able to account for viscoelastic, thermoelastic and radiation losses in materials. ${ }^{1}$

In this paper (Part II, Sec. II) the equations of motion for the damped plate are briefly reviewed in the general case. For convenience, these equations are written in the Laplace domain with complex rigidity factors. The system is complemented by initial and boundary conditions. The initial impact is modeled by Hertz's law of contact. Most of the presented results are obtained in the case of freely suspended plates. However, the model is able to account for other simple boundary conditions, such as clamped or simply supported plates.

The main purpose of this paper is to validate a vibrational model for the impacted plate. No attempt is made to compute the complete acoustic field around the plate with great accuracy. However, in order to facilitate the comparison with real sounds, rather than with vibrational quantities (such as velocity or acceleration at a given point of the plate), a simple radiation model is to calculate radiation from the plate displacement, in order to obtain simulated waveforms having the same dimension as recorded sounds.

In Sec. III, the equations of the model are put into a

\footnotetext{
${ }^{a)}$ Present address: 64 rue des Moines, 75017 Paris, France.

b) Present address: ENSTA-UME, Chemin de la Hunière 91761 Palaiseau cedex, France, electronic mail: chaigne@ensta.fr
}

numerical form using an explicit finite-difference scheme of second order in time and fourth order in space. Similar schemes were successfully applied in the past to the coupling between plate vibration and acoustic radiation by Frendi et $a .^{2}$ and by Schedin et al. for the time-domain simulation of undamped isotropic plates subjected to impacts. ${ }^{3}$ The numerical formulation is followed by a necessary analysis of stability and dispersion properties, since no previous references were found in the literature for similar problems which included damping.

The results presented in Sec. IV show the efficiency of the simulation. Following the mode of presentation previously used for xylophones, ${ }^{4}$ the method is first validated by comparisons between analytical and numerical results in simple situations and, second, by comparisons between measurements and simulations for damped rectangular plates made either of isotropic or orthotropic materials.

\section{THE GOVERNING EQUATIONS}

\section{A. The damped-plate equations}

The flexural vibrations of a rectangular Kirchhoff-Love plate are considered here (see Fig. 1 for the geometry of the problem). The equations are written in the orthotropic case. The losses are expressed by means of a differential operator similar to the one used in viscoelasticity. For a plate of small thickness $h$, the transverse displacement $W(x, y, t)$, as a function of the coordinates $x, y$ and time $t$, is governed by the following equations (expressed in the Laplace domain with Laplace variable $s):{ }^{1,5}$ 


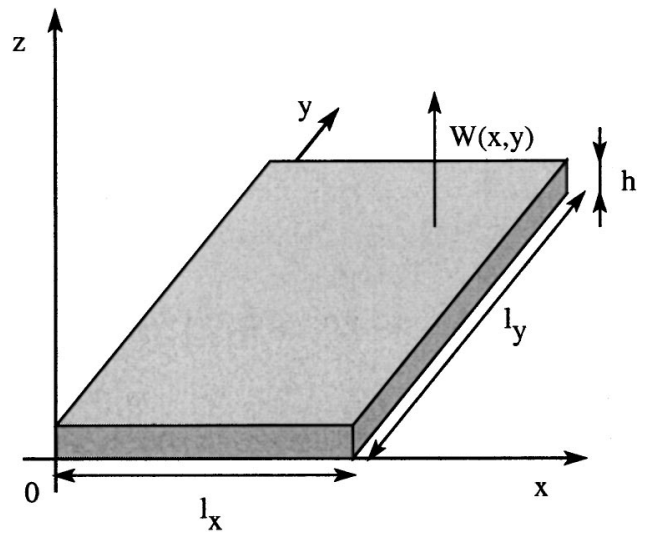

FIG. 1. Geometry of the plate of dimensions $l_{x}$ and $l_{y}$ and of thickness $h$. The flexural displacement $W(x, y)$ is oriented along the $z$ axis.

$$
\begin{aligned}
\left(\begin{array}{c}
\tilde{M}_{x}(x, y, s) \\
\tilde{M}_{y}(x, y, s) \\
\tilde{M}_{x y}(x, y, s)
\end{array}\right)= & -h^{3}\left(\begin{array}{ccc}
\widetilde{D}_{1}(s) & \widetilde{D}_{2}(s) / 2 & 0 \\
\widetilde{D}_{2}(s) / 2 & \widetilde{D}_{3}(s) & 0 \\
0 & 0 & \widetilde{D}_{4}(s) / 2
\end{array}\right) \\
& \times\left(\begin{array}{c}
\widetilde{W}_{, x x}(x, y, s) \\
\widetilde{W}_{, y y}(x, y, s) \\
\widetilde{W}_{, x y}(x, y, s)
\end{array}\right), \\
\rho h\left(s^{2}+R_{f} s\right) \widetilde{W}(x, y, s)= & \widetilde{M}_{x, x x}(x, y, s)+\widetilde{M}_{y, y y}(x, y, s) \\
& +2 \widetilde{M}_{x y, x y}(x, y, s)+\widetilde{f}_{z}(x, y, s),
\end{aligned}
$$

where the subscripts , $x x, y y$ and , $x y$ denote the partial derivatives of the variables. The first three equations correspond to the viscoelasticlike strain-stress relationships for thin orthotropic plates. $\tilde{M}_{x}(x, y, s), \quad \tilde{M}_{y}(x, y, s)$ and $\widetilde{M}_{x y}(x, y, s)$ are the Laplace transforms of the bending and twisting moments and $\widetilde{D}_{i}(s)$ are the four complex rigidities. $\widetilde{W}(x, y, s)$ is the Laplace transform of the displacement. The fourth equation in Eq. (1) derives from Newton's second law, where $\rho$ is the density of the material, $R_{f}$ a viscous damping coefficient, and $f_{z}(x, y, t)$ an excitation source term.

The finite-difference formulation of the problem is facilitated by expressing the strain-stress relationships in terms of differential equations. In the present model, the complex rigidities are written in the form:

$$
\widetilde{D}_{i}(s)=D_{i}\left(1+\widetilde{d}_{i}(s)\right)=D_{i} \frac{1+\sum_{v=1}^{N} s^{v} p_{i v}}{1+\sum_{w=1}^{N} s^{w} q_{w}},
$$

where the coefficients $p_{i v}$ and $q_{w}$ depend on the damping mechanisms (see Table II). The $D_{i}$ correspond to the static rigidities of the plate. The $\widetilde{d}_{i}$ are perturbation terms due to damping. In order to ensure that the model is dissipative the coefficients $p_{i v}$ and $q_{w}$ must fulfill a number of constraints. ${ }^{1}$

\section{B. The boundary conditions}

For a plate of finite size, Eq. (1) must be completed by the boundary conditions. Only three ideal conditions for edges parallel to the axes of symmetry of the material are considered here. For the edges located at $x=0$ and $x=l_{x}$ (see Fig. 1), these conditions are ${ }^{6}$

- for a free edge $(\mathrm{F})$ :

$$
M_{x}=0 \text { and } \frac{\partial M_{x}}{\partial x}+2 \frac{\partial M_{x y}}{\partial y}=0
$$

- for a simply supported edge (SS):

$$
W=0 \text { and } M_{x}=0 \text {, }
$$

- for a clamped edge (C):

$$
W=0 \text { and } \frac{\partial W}{\partial x}=0 .
$$

Similar conditions are given for the edges located at $y$ $=0$ and $y=l_{y}$ by interchanging $x$ and $y$ in Eqs. (3)-(5).

\section{The interaction between the plate and the impactor}

In Eq. (1), it is assumed that the load $f(x, y, t)$ due to the interaction between the plate and the impactor is governed by Hertz's law of contact. ${ }^{7}$ This method has been used with success in the past for the time-domain modeling of impacted xylophone bars $^{8}$ and for a mixed time-frequency investigation on impacts on plates. ${ }^{9}$ The motion $W_{i}$ of the impactor is a solution of the following system:

$$
\begin{aligned}
& f(x, y, t)=g\left(x-x_{0}, y-y_{0}\right) F_{H}(t), \\
& F_{H}(t)=\left\{\begin{array}{c}
{\left[\left(W\left(x_{0}, y_{0}, t\right)-W_{i}(t)\right) / k_{H}\right]^{3 / 2}} \\
\text { if } W_{i}(t)<W\left(x_{0}, y_{0}, t\right), \\
0 \quad \text { otherwise }
\end{array}\right. \\
& \frac{d^{2} W_{i}(t)}{d t^{2}}=\frac{F_{H}(t)}{m_{i}},
\end{aligned}
$$

where $g\left(x-x_{0}, y-y_{0}\right)$ is a normalized spatial window centered at the impact point, $F_{H}$ is the interaction force, $W$ $-W_{i}$ is the relative displacement between the impactor and the plate at the impact point, $m_{i}$ is the mass of the impactor and $k_{H}$ is Hertz's constant which depends on the surface geometries and elastic properties of the two bodies in contact. In Eq. (6), the vibrations in the impactor are neglected and it is assumed that the contact surface remains constant during the impact. These assumptions are reasonable when the dimensions of the impactor are small compared to those of the plate. ${ }^{9}$

\section{The radiation equation}

For the purpose of simplicity, only the case of baffled plates will be considered here. Thus, for computing the radiated pressure from the plate displacement $W$, the following time-domain formulation of the Rayleigh integral was used:

$$
p(\mathbf{r}, t)=-\frac{\rho_{a}}{2 \pi} \iint_{S_{0}} \frac{1}{\left|\mathbf{r}-\mathbf{r}_{0}\right|} \frac{\partial^{2} W}{\partial t^{2}}\left(\mathbf{r}_{0}, t-\frac{\left|\mathbf{r}-\mathbf{r}_{0}\right|}{c}\right) d S_{0},
$$

where $p$ is the sound pressure at the position $\mathbf{r}$ in the sound field, $\rho_{a}$ is the air density, $c$ is the speed of sound in air, $S_{0}$ is the surface of the plate and $\mathbf{r}_{\mathbf{0}}$ refers to the position of the source points on the plate surface. 


\section{THE NUMERICAL MODEL}

The present work is limited to rectangular plates for which the boundaries coincide with the nodes of a regular rectangular grid. For this class of geometries, it is known that the Finite-Difference Methods (FDM) are convenient for solving vibration problems in the time domain. ${ }^{10,11}$

In this section, the use of a 2-4 finite-difference scheme for solving the impacted plate equations is studied in terms of stability and numerical dispersion. This scheme is of second order in time and fourth order in space. The numerical parameters to be determined are the time step $\Delta t$ and the two spatial steps $\Delta x, \Delta y$ along the $x$ and $y$ axis, respectively. The appropriate selection of these parameters is imposed both by the numerical stability condition and by the order of accuracy required for the dispersion.

In what follows, the guideline for the dispersion criteria was given by the accuracy required in the context of audio applications. According to Moore, ${ }^{12}$ the minimum value of the relative difference limen for pitch of a pure tone is about $0.5 \%$ at $2 \mathrm{kHz}$, and becomes greater than $5 \%$ for frequencies above $5 \mathrm{kHz}$. Since there are no available data for the difference limen relative to tones made of simultaneous inharmonic frequencies, the pure tone difference limen was used as reference for the required accuracy of the numerical model.

\section{A. Explicit finite-difference schemes}

The transverse displacement of the plate is computed at the nodes $(x=l \Delta x, y=m \Delta y, t=n \Delta t)$ of a rectangular grid. The value of a field variable $v(x, y, t)$ expressed on this mesh is denoted $v_{l, m}^{n}=v(l \Delta x, m \Delta y, n \Delta t)$.

The elastic and inertial terms, involving second order partial derivatives versus time or space, are discretized by means of centered finite difference operators (see Appendix A). The damping terms are approximated by decentered operators which has the advantage to keep the explicit character of the numerical formulation. This strategy is justified by the fact that the damping terms $\widetilde{d}_{i}$ defined in Eq. (2) are assumed to be first order correction terms. Centered operators for the damping terms would have led to an implicit scheme. The time derivatives in the damping terms $\widetilde{d}_{i}$ are approximated by backward difference operators which, in terms of the z-transform, amounts to replacing the continuous Laplace variable $s$ by the discrete operator $\left(1-z^{-1}\right) / \Delta t$. Thus, the discrete approximation of $\widetilde{d}_{i}$, written in descending power of $z$, is written:

$$
\frac{\sum_{r=0}^{N} \chi_{i r} z^{-r}}{1+\sum_{r=1}^{N} \psi_{r} z^{-r}}=\frac{\Delta t^{N}+\sum_{r=1}^{N} \Delta t^{N-r}\left(1-z^{-1}\right)^{r} p_{i r}}{\Delta t^{N}+\sum_{r=1}^{N} \Delta t^{N-r}\left(1-z^{-1}\right)^{r} q_{r}} .
$$

In Eq. (8), the constants $\chi_{i r}$ and $\psi_{r}$ are obtained by identifying term by term the two sides of Eq. (8).

The finite-difference approximation of Eq. (1) is then given by:

$$
\begin{aligned}
\left(M_{x}\right)_{l, m}^{n}= & -h^{3} D_{1} \sum_{r=0}^{N} \chi_{1 r}\left(D_{x x} W\right)_{l, m}^{n-r} \\
& -h^{3} \frac{D_{2}}{2} \sum_{r=0}^{N} \chi_{2 r}\left(D_{y y} W\right)_{l, m}^{n-r}-\sum_{r=1}^{N} \psi_{r}\left(M_{x}\right)_{l, m}^{n-r} \\
\left(M_{y}\right)_{l, m}^{n}= & -h^{3} D_{3} \sum_{r=0}^{N} \chi_{3 r}\left(D_{y y} W\right)_{l, m}^{n-r} \\
& -h^{3} \frac{D_{2}}{2} \sum_{r=0}^{N} \chi_{2 r}\left(D_{x x} W\right)_{l, m}^{n-r}-\sum_{r=1}^{N} \psi_{r}\left(M_{y}\right)_{l, m}^{n-r} \\
\left(M_{x y}\right)_{l+1 / 2, m+1 / 2}^{n}=-h^{3} \frac{D_{4}}{2} \sum_{r=0}^{N} \chi_{4 r}\left(D_{x y} W\right)_{l+1 / 2, m+1 / 2}^{n-r} & -\sum_{r=1}^{N} \psi_{r}\left(M_{x y}\right)_{l+1 / 2, m+1 / 2}^{n-r} \\
W_{l, m}^{n+1}= & \left(2-R_{f} \Delta t\right) W_{l, m}^{n}+\left(R_{f} \Delta t-1\right) W_{l, m}^{n-1} \\
+ & \frac{\Delta t^{2}}{\rho h}\left(\left(D_{x x} M_{x}\right)_{l, m}^{n}+\left(D_{y y} M_{y}\right)_{l, m}^{n}\right. \\
+ & \left.2\left(\widetilde{D}_{x y} M_{x y}\right)_{l, m}^{n}+\left(f_{z}\right)_{l, m}^{n}\right),
\end{aligned}
$$

where the discrete spatial operators $D_{x x}, D_{y y}, D_{x y}$ and $\widetilde{D}_{x y}$ are fourth order approximations (see Appendix A). It can be seen from Eq. (9) that the transverse displacement $W$ is recursively determined at time $n+1$ from its values at previous time steps.

\section{B. Boundary conditions}

The numerical approximation of the boundary conditions is obtained by means of the image method. It consists of replacing the boundaries by virtual sources located at points external to the plate. In what follows, only the boundary conditions for a free straight edge located at $x=0$, for a semi-infinite plate, is discussed [see Eq. (3)]. The results can be easily extended to simply supported and clamped edges. ${ }^{13}$ The numerical formulation of the boundary conditions described below is conducted so as to maintain the overall accuracy of the scheme and to limit the computational domain.

It is assumed that the semi-infinite plate is located in $x$ $>0$ (see Fig. 1) and that the transverse displacement $W$, at time $n \Delta t$ and at every previous time step, is known at each point $l \Delta x, m \Delta y$ such that $l \geqslant-1$. The values of $W$ at time $n+1$ are then obtained by using the following procedure:

- First, the bending and twisting moments are computed at time $n \Delta t$. This computation is performed by applying the first three equations in Eq. (9) to each point of the grid for which $x>0$. For $x=0, M_{x}$ is equal to zero and $M_{y}$ is derived from the displacement by using the second equation in Eq. (9), after replacing $D_{x x}^{(4)}$ by the second order operator $D_{x x}^{(2)}$ in order to limit the spatial extension of the boundary domain. $\left(M_{x y}\right)_{-1 / 2, m}^{n}$ is given by the third equation in Eq. (9) where $D_{x y}^{(4)}$ is replaced by the operator $D_{x}^{(2)} o D_{y}^{(4)}$ defined as: 


$$
\begin{aligned}
\left(D_{x}^{(2)} o D_{y}^{(4)} W\right)_{-1 / 2, m+1 / 2}^{n} & \\
= & \frac{1}{24 \Delta x \Delta y}\left[\left(W_{0, m-2}^{n}-27 W_{0, m-1}^{n}\right.\right. \\
& \left.+27 W_{0, m}^{n}-W_{1, m+2}^{n}\right)-\left(W_{-1, m-2}^{n}-27 W_{-1, m-1}^{n}\right. \\
& \left.\left.+27 W_{-1, m}^{n}-W_{-1, m+2}^{n}\right)\right] .
\end{aligned}
$$

- The application of the image method also involves the computation of the values of $M_{x}$ at points $x=-\Delta x$. This is performed by considering the second condition in Eq. (3), for which a third order decentered approximation in $\Delta x$ and fourth order in $\Delta y$ leads to:

$$
\begin{aligned}
\left(M_{x}\right)_{-1, m}^{n}= & 3\left(M_{x}\right)_{1, m}^{n}-\frac{1}{2}\left(M_{x}\right)_{2}^{n}-3 \Delta x\left(\left(\widetilde{D}_{y}^{(4)} M_{x y}\right)_{-1 / 2, m}^{n}\right. \\
& \left.+\left(\widetilde{D}_{y}^{(4)} M_{x y}\right)_{1 / 2, m}^{n}\right)
\end{aligned}
$$

where

$$
\begin{aligned}
\left(\widetilde{D}_{y}^{(4)} M_{x y}\right)_{l+1 / 2, m}^{n}= & \frac{9}{8 \Delta y}\left(\left(M_{x y}\right)_{l+1 / 2, m+1 / 2}^{n}-\left(M_{x y}\right)_{l+1 / 2, m-1 / 2}^{n}\right) \\
& -\frac{1}{24 \Delta y}\left(\left(M_{x y}\right)_{l+1 / 2, m+3 / 2}^{n}\right. \\
& \left.-\left(M_{x y}\right)_{l+1 / 2, m-3 / 2}^{n}\right)
\end{aligned}
$$

- The final task consists in computing $W$ at time ( $n$ $+1) \Delta t$. This is performed by using the fourth equation in Eq. (9) for the points $l>0$, and by replacing the fourth order operators in $\Delta x$ by second order ones in this equation at points $l=0$. The displacement at the image points $l=-1$ is derived from the free edge condition $M_{x}=0$ at $x=0$, for which a second order in $\Delta x$, and fourth order in $\Delta y$ approximation leads to the following expression of $W_{-1, m}^{n+1}$ :

$$
W_{-1, m}^{n+1}=-\frac{\Delta x^{2} D_{2}}{2 D_{1}}\left(D_{y y}^{(4)} W\right)_{0, m}^{n+1}+2 W_{0, m}^{n+1}-W_{1, m}^{n+1} .
$$

The influence of the damping terms is neglected in the discrete approximation of the boundary conditions. The schemes for the three other edges of the plate obey the same principles as the one presented above for $x=0$.

\section{Stability}

The numerical parameters of explicit difference schemes must fulfill a stability condition which guarantees the convergence of the numerical solution. ${ }^{14}$ In this paragraph, the stability condition for the 2-4 scheme applied to the undamped plate model is presented. The results are then extended to the damped model. A distinction is made in what follows between two definitions of stability: ${ }^{14}$

- The numerical scheme is called weakly stable if the solution at a fixed time $T=n \Delta t$ is bounded for all $\Delta t$ with respect to the Euclidean norm $\left\|W^{n}\right\|=\left(\Sigma_{l, m}\left|W_{l, m}^{n}\right|^{2}\right)^{1 / 2}$.

- The scheme is called strongly stable if the solution remains bounded for all $T$.

A strong stability condition can be obtained for the undamped model. In the damped case, paradoxically, only a weak stability condition can be given.

\section{Undamped model}

In this paragraph, the fluid damping constant $R_{f}$ and the damping coefficients $p_{i r}$ and $q_{r}$ are set equal to zero. The Fourier method is used for determining the stability criterion. ${ }^{14}$ It consists of studying the modulus of one particular solution $W_{l, m}^{n}=w_{n} \exp \left(j k_{x} l \Delta x+j k_{y} m \Delta y\right)$ of the homogeneous equation. Injecting $W_{l, m}^{n}$ in Eq. (9) for $f_{l, m}^{n}=0$ leads to the following equation for $w_{n}$ :

$$
w_{n+1}+\left(b\left(k_{x}, k_{y}\right)^{2} \Delta t^{2}-2\right) w_{n}+w_{n-1}=0
$$

with:

$$
\begin{aligned}
b\left(k_{x}, k_{y}\right)^{2}= & 16\left(\frac{\xi_{1}^{2}}{\Delta x^{4}}\left(X^{2}+\frac{1}{3} X^{4}\right)^{2}+\frac{\xi_{3}^{2}}{\Delta y^{4}}\left(Y^{2}+\frac{1}{3} Y^{4}\right)^{2}\right. \\
& +\frac{\xi_{2}^{2}}{\Delta x^{2} \Delta y^{2}}\left(X^{2}+\frac{1}{3} X^{4}\right)\left(Y^{2}+\frac{1}{3} Y^{4}\right) \\
& \left.+\frac{\xi_{4}^{2}}{\Delta x^{2} \Delta y^{2}}\left(X+\frac{1}{6} X^{3}\right)^{2}\left(Y+\frac{1}{6} Y^{3}\right)^{2}\right)
\end{aligned}
$$

and where

$$
\xi_{i}=\sqrt{\frac{h^{2} D_{i}}{\rho}}, \quad X=\sin \frac{k_{x} \Delta x}{2}, \quad Y=\sin \frac{k_{y} \Delta y}{2} .
$$

The stability criterion is satisfied if the discriminant of the characteristic polynomial associated with Eq. (14) is negative for all $k_{x}$ and $k_{y}$. This leads to the following condition:

$$
\Delta t \leqslant \frac{1}{2 \sqrt{\left(\frac{4}{3}\right)^{2}\left(\xi_{1}^{2}+\frac{\xi_{2}^{2}}{r_{1}^{2}}+\frac{\xi_{3}^{2}}{r_{1}^{4}}\right)+\left(\frac{7}{6}\right)^{4} \frac{\xi_{4}^{2}}{r_{1}^{2}}}},
$$

where $r_{1}=\Delta y / \Delta x$.

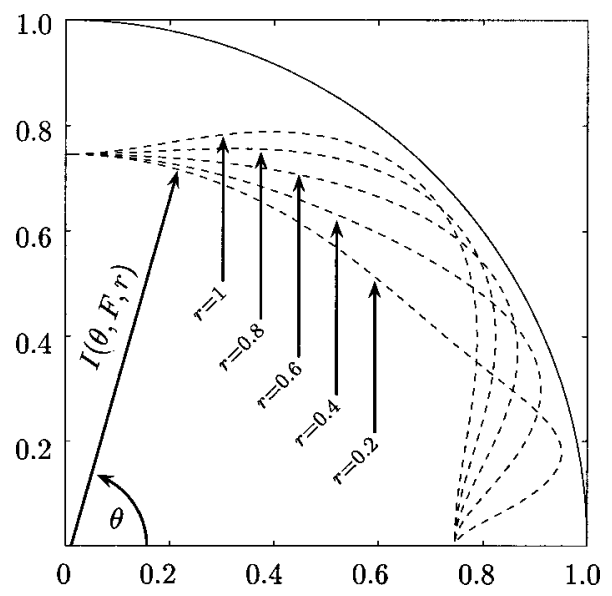

FIG. 2. Numerical isotropy $I(\theta, F, r)$ for a reduced frequency $F$ equal to 0.2 and for different values of the degree $r$ of anisotropy: $r$ $=\{0.2,0.4,0.6,0.8,1\}$. 
(a)
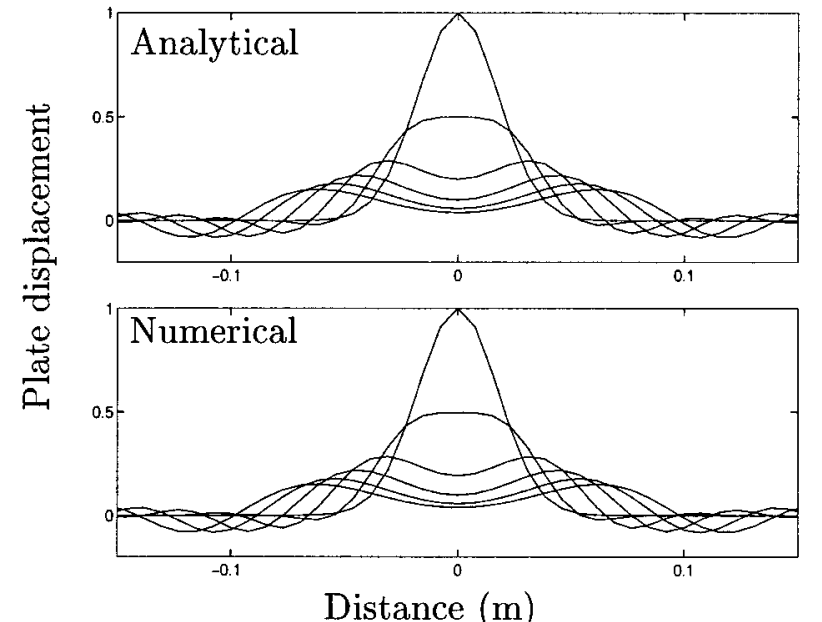

Distance (m)

(b)

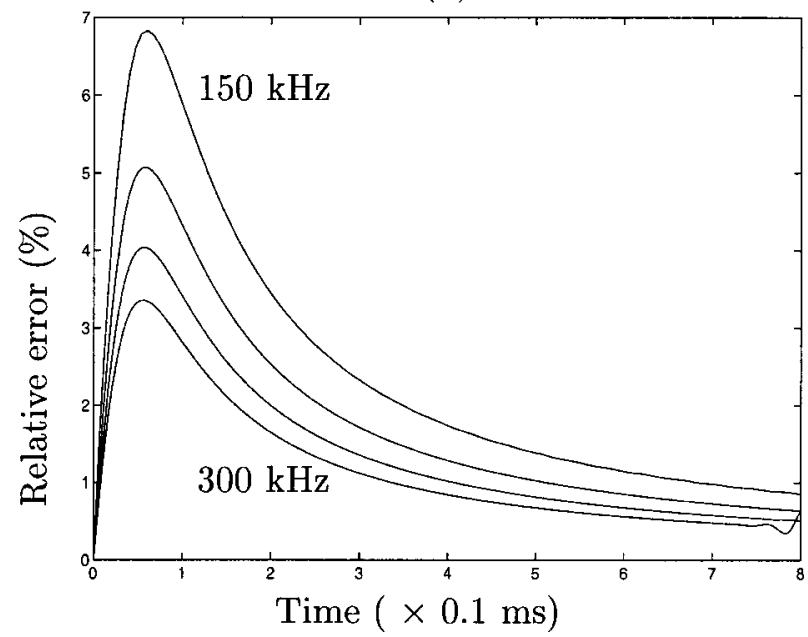

FIG. 3. (a) Infinite isotropic plate profile at successive instants of time $t$ $=\{0,0.05,0.10,0.15,0.20,0.25\} \mathrm{ms}$. Top: analytical solution. Bottom: numerical solution. (b) Relative error (in \%) between analytical and numerical solutions versus time for four different sampling frequencies $f_{e}$ $=\{150,200,250,300\} \mathrm{kHz}$.

\section{Damped model}

A model including the damping terms is now considered. To facilitate the presentation, the case of a damped bending bar (1D problem) is first presented and the results are then extended to plates (2D).

Similarly to Eq. (1), the equations governing the transverse displacement $W(x, t)$ of a thin bar are written:

$$
\begin{gathered}
\widetilde{M}_{x}=-E I \frac{1+\sum_{r=1}^{N} p_{r} s^{r}}{1+\sum_{r=1}^{N} q_{r} s^{r}} \widetilde{W}_{, x x} \\
\rho S\left(s^{2}+R_{f} s\right) \widetilde{W}=\widetilde{M}_{x, x x}+\widetilde{f}_{z} .
\end{gathered}
$$

The corresponding FD scheme is written:

$$
\begin{aligned}
\left(M_{x}\right)_{l}^{n}= & -I E \sum_{r=0}^{N} \chi_{r}\left(D_{x x} W\right)_{l}^{n-r}-\sum_{r=1}^{N} \psi_{r}\left(M_{x}\right)_{l}^{n-r}, \\
W_{l}^{n+1}= & \left(2-R_{f} \Delta t\right) W_{l}^{n}+\left(R_{f} \Delta t-1\right) W_{l}^{n-1} \\
& -\frac{\Delta t^{2}}{\rho S}\left(\left(D_{x x} M_{x}\right)_{l}^{n}+\left(f_{z}\right)_{l}^{n}\right),
\end{aligned}
$$

where $E$ is Young's modulus, $I$ is the geometrical moment and $S$ is the cross-section of the bar. The term $f_{z}$ denotes the external force density by unit length. $D_{x x}$ is a fourth order discrete operator.

A necessary condition for weak stability is obtained by studying the amplification matrix ${ }^{14}$ (see Appendix B). This condition is written:

$$
\Delta x^{2} \geqslant 2 \Delta t \sqrt{\frac{I E p_{N}}{\rho S q_{N}}}
$$

It is found that the condition expressed in Eq. (20) is similar to the one obtained with the Fourier method in the undamped case, except that $E$ is now replaced by $E p_{N} / q_{N}$. This term corresponds to the high frequencies' asymptotic value of the complex Young's modulus.

The stability condition for the 2D system Eq. (9) is determined in the same way as for the 1D case (see Appendix B). It is found that the weak stability condition of the damped 2-4 scheme is similar to Eq. (17), after replacing each $D_{i}$ by $D_{i} p_{i N} / q_{N}$. Since $p_{i N} / q_{N}$ is always greater than unity for a dissipative model, this result shows that the stability condition is more restrictive when damping terms are taken into account than for the undamped plate. However, for the investigated materials (aluminum, steel, glass and wood), it has been found that the consecutive increase in size of the minimum space step remains below a few percent, compared to the undamped case.

\section{Accuracy}

One method for estimating the deviation between exact and approximate solutions consists of calculating the difference between continuous and discrete frequencies. ${ }^{10,15}$ For simplicity, only the undamped model will be discussed here.

Inserting the solution $W(x, y, t)=\left(A e^{j \omega t}\right.$ $\left.+B e^{-j \omega t}\right) \exp \left(j k_{x} x+j k_{y} y\right)$ in Eq. (1), without damping terms and for $f_{z}=0$, yields the continuous dispersion equation:

$$
k=\sqrt{\frac{\omega}{\xi_{\theta}}} \quad \text { with } k=\sqrt{k_{x}^{2}+k_{y}^{2}}, \quad \theta=\arctan \frac{k_{y}}{k_{x}}, \quad \xi_{\theta}=\frac{h^{2}}{\rho}\left(D_{1} \cos ^{4} \theta+\left(D_{2}+D_{4}\right) \cos ^{2} \theta \sin ^{2} \theta+D_{3} \sin ^{4} \theta\right) .
$$


(a)
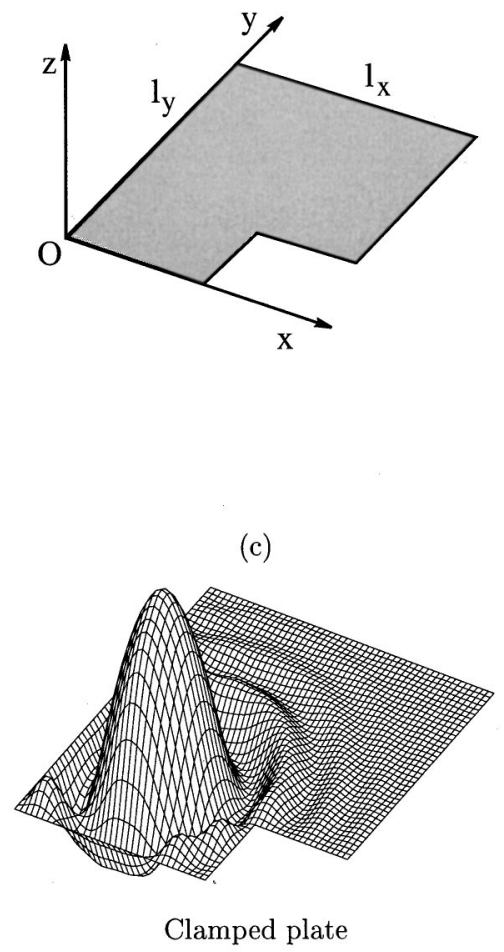

(b)

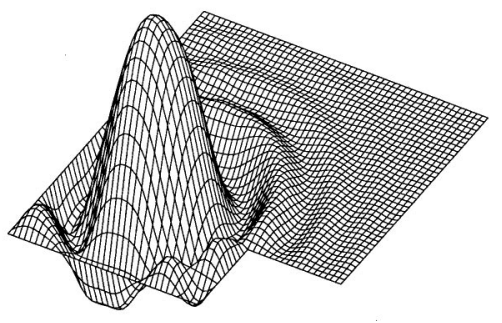

Simply supported plate

(d)

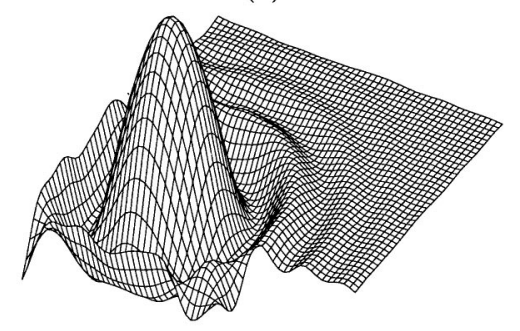

FIG. 4. Bending vibrations of a L-shaped isotropic plate, a few ms after the impact, with different boundary conditions. (a) Geometry of the plate at rest. (b) Simply supported plate. (c) Clamped plate. (d) Free plate.
Similarly, provided that the stability condition is verified, the introduction of the component $W_{l, m}^{n}=\left(A e^{j \omega_{\text {num }} n \Delta t}\right.$ $\left.+B e^{-j \omega_{\text {num }} n \Delta t}\right) \exp \left(j k_{x} l \Delta x+j k_{y} m \Delta y\right)$ in Eq. (9) leads to the numerical dispersion equation:

$$
\omega_{\text {num }}=\frac{2}{\Delta t} \arcsin \frac{b\left(k_{x}, k_{y}\right) \Delta t}{2},
$$

where $b\left(k_{x}, k_{y}\right)$ is defined in Eq. (15).

In order to simplify the mathematical derivations, it is assumed here that $\xi_{2}=0$ and that $\xi_{4}=\sqrt{2 \xi_{1} \xi_{3}}$ which constitutes a reasonable approximation for a large class of orthotropic materials. ${ }^{16}$ This allows us to characterize the anisotropy of the material with only one parameter $r=\xi_{3} / \xi_{1}$. Under these conditions Eqs. (21) and (22), combined with Eq. (17), yield:

$$
\begin{aligned}
& \Omega_{\text {num }}= 2 \arcsin \left[\frac { 3 6 } { \sqrt { 9 4 1 0 } } \left(\left(X^{2}+\frac{X^{4}}{3}\right)^{2}+\left(Y^{2}+\frac{Y^{4}}{3}\right)^{2}\right.\right. \\
&\left.\left.+2\left(X+\frac{X^{3}}{6}\right)^{2}\left(Y+\frac{Y^{3}}{6}\right)^{2}\right)^{1 / 2}\right] \\
& X=\sin \left(\left(\frac{4705}{2592}\right)^{1 / 4} \frac{\sqrt{\Omega} \cos \theta}{\sqrt{\cos ^{2} \theta+r^{2} \sin ^{2} \theta}}\right) \\
& Y=\sin \left(\left(\frac{4705}{2592}\right)^{1 / 4} \frac{r \sqrt{\Omega} \sin \theta}{\sqrt{\cos ^{2} \theta+r^{2} \sin ^{2} \theta}}\right),
\end{aligned}
$$

where $\Omega=\omega \Delta t=2 \pi F$ and $\Omega_{\text {num }}=\omega_{\text {num }} \Delta t$ are the reduced angular frequencies.

Finally, the relative error in frequency is given by:

$$
e(\Omega, \theta)=\frac{\Omega-\Omega_{\text {num }}}{\Omega} .
$$

It has been observed that about 15 time steps per period are required with the 2-4 scheme in order to keep the error in frequency smaller than $5 \%$ at $20 \mathrm{kHz}$, whereas more than 40 time-steps by period would have been needed with the 2-2 scheme. In the audio range $0-20 \mathrm{kHz}$, this degree of accuracy requires a sampling rate approximately equal to $300 \mathrm{kHz}$ for the $2-4$ scheme, whereas $800 \mathrm{kHz}$ would have been needed with the 2-2 scheme.

For a material with a given anisotropy degree $r$, Eq. (23) shows that the numerical error not only depends on frequency, but also on the propagation angle $\theta$ in the plate. A convenient method for representing this error is to define an isotropy index: ${ }^{15}$

$$
I(\theta, F, r)=\frac{c_{\text {num }}}{c},
$$

where $c_{\text {num }}$ is the numerical phase velocity and $c$ the exact phase velocity. Figure 2 represents the variations of $I$ with $\theta$, for a given reduced frequency $F=0.2$, and for different values of the degree $r$ of anisotropy. Notice that the angle $\theta_{m}$ corresponding to the maximum index $I_{M}$ moves from $\pi / 4$ to zero as the anisotropy degree $r$ of the material decreases. However, the ratio $I_{M} / I_{m}$ between the maximum and the minimum values of the isotropy index remains unchanged as $r$ varies.

In our applications, the plate equation has been solved with a 2-4 scheme and with a sampling rate of $192 \mathrm{kHz}$. This leads to an error of $8 \%$ in the estimation of the phase velocity at $20 \mathrm{kHz}$ and of $2.5 \%$ at $10 \mathrm{kHz}$. 
(a)

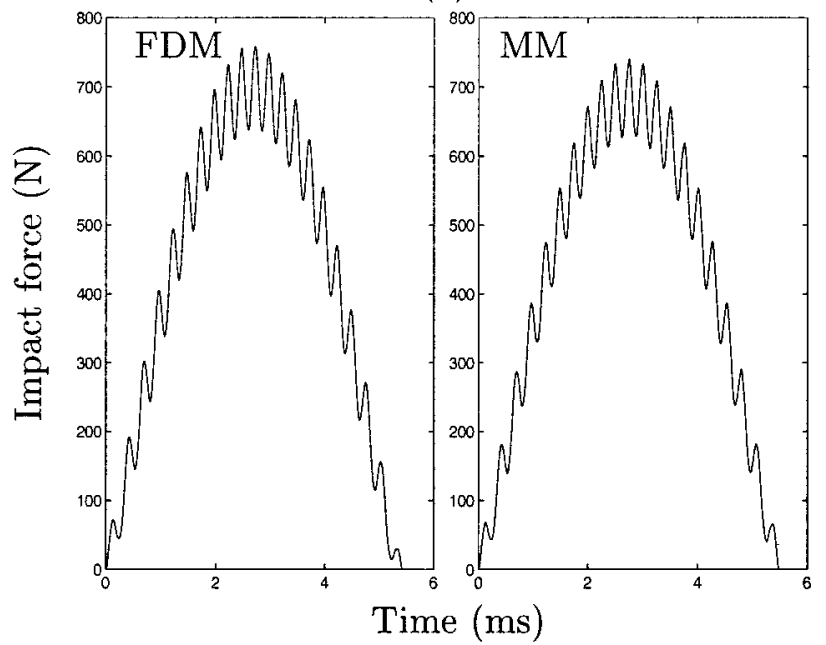

(b)

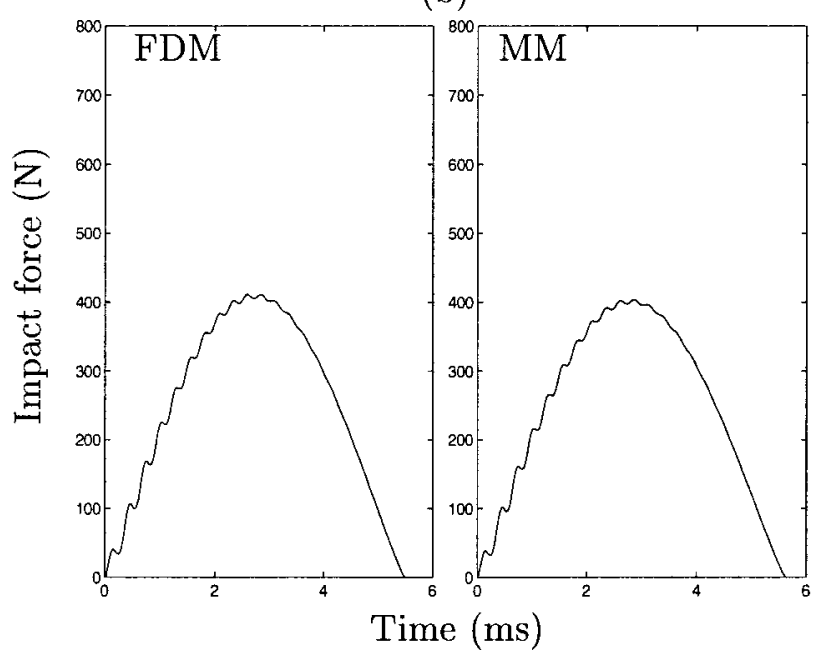

FIG. 5. Interaction force between an orthotropic plate and an impactor during the contact time, for two different initial velocities of the impactor: (a) $1.45 \mathrm{~m} / \mathrm{s}$, (b) $0.6 \mathrm{~m} / \mathrm{s}$. Left: Simulation with the present Finite-Differences Method (FDM). Right: Simulation made by McMillan (Ref. 9).

\section{E. Computation of the plate-impactor interaction}

The discrete formulation of Eq. (6) is given by

$$
\begin{aligned}
& f_{l, m}^{n}=-g_{l, m} F_{h}^{n} \\
& W_{i}^{n+1}=2 W_{i}^{n}-W_{i}^{n-1}+\frac{\Delta t^{2}}{m_{s}} F_{h}^{n} \\
& F_{h}^{n+1}= \begin{cases}{\left[\left(W_{p}^{n+1}-W_{i}^{n+1}\right) / k_{H}\right.} & ]^{3 / 2} \text { if } W_{i}^{n+1}<W_{p}^{n+1} \\
0 & \text { otherwise. }\end{cases}
\end{aligned}
$$

$W_{p}$ is the displacement of the plate at impact point $\left(x_{0}, y_{0}\right)$. The spatial window $g_{l, m}$ is a triangular function of width $2 \Delta x$ and $2 \Delta y$, centered at point $\left(x_{0}, y_{0}\right)$.

\section{F. Computation of the sound pressure}

The semi-discrete formulation of the Rayleigh integral presented in Eq. (7) is obtained by using a standard trapezoidal rule:

$$
\begin{aligned}
p(\mathbf{r}, t)= & -\frac{\rho_{a}}{2 \pi} \sum_{l=0}^{N_{x}-1} \sum_{m=0}^{N_{y}-1} \frac{\Delta x \Delta y}{4} \frac{d^{2}}{d t^{2}}\left(\frac{W_{l, m}\left(t-R_{l, m} / c\right)}{R_{l, m}}\right. \\
& +\frac{W_{l+1, m}\left(t-R_{l+1, m} / c\right)}{R_{l+1, m}}+\frac{W_{l, m+1}\left(t-R_{l, m+1} / c\right)}{R_{l, m+1}} \\
& \left.+\frac{W_{l+1, m+1}\left(t-R_{l+1, m+1} / c\right)}{R_{l+1, m+1}}\right) .
\end{aligned}
$$

In this equation, $N_{x}$ and $N_{y}$ are the nearest integers smaller than or equal to $l_{x} / \Delta x$ and $l_{y} / \Delta y$, respectively, where $0<x<l_{x}$ and $0<y<l_{y}$ is the plate domain. $R_{l, m}$ is the distance between the listening point and each mesh point of the plate and $R_{l, m} / c$ is the corresponding time delay. After time-domain discretization, this quantity is approximated by the nearest multiple of $\Delta t$ such that:

$$
W_{l, m}\left(n \Delta t-R_{l, m} / c\right) \simeq W_{l, m}^{n-i_{l, m}} \quad \text { with } \quad i_{l, m}=\operatorname{int}\left(\frac{R_{l, m}}{c \Delta t}\right) .
$$

Finally, the discrete formulation of Eq. (27) is written:

$$
p(\mathbf{r}, n \Delta t)=p^{n}=D_{t t} \sum_{i=i_{\min }}^{i_{\max }} \sum_{l=0}^{N_{x}} \sum_{m=0}^{N_{y}} \delta_{i i_{l, m}} A_{l, m} W_{l, m}^{n-i},
$$

where

$$
i_{\min }=\min \left(i_{l, m}\right), \quad i_{\max }=\max \left(i_{l, m}\right)
$$

$D_{t t}$ discrete backward time-derivative operator

$$
\begin{aligned}
A_{l, m}= & -\frac{\Delta x \Delta y \rho_{a}}{2 \pi R_{l, m}} B_{l, m} \\
B_{l, m}= & \begin{cases}1 \quad & \text { if } 0<l<N_{x} \text { and } 0<m<N_{y} \\
1 / 2 & \text { if }\left(\left(l=0 \text { or } N_{x}\right) \text { and }\left(0<m<N_{y}\right)\right) \\
& \text { or }\left(\left(m=0 \text { or } N_{y}\right) \text { and }\left(0<l<N_{x}\right)\right) \\
1 / 4 & \text { otherwise. }\end{cases}
\end{aligned}
$$

\section{RESULTS OF SIMULATIONS}

In order to assess the validity of the method, this section starts with a comparison between the numerical result and the theoretical solution obtained in a simple case where the plate displacement is given analytically. This presentation is followed by the simulation of the plate for three different boundary conditions. The force pulse obtained with our numerical scheme is then compared to the force pulse calculated for the same plate impacted with the same impactor using the method developed by McMillan. ${ }^{9}$ Finally, simulated waveforms, and their corresponding spectra, are compared to measurements for four different materials (aluminum, glass, carbon fiber and wood).

\section{A. Comparison with analytical displacement}

An analytical solution to the plate equation can be obtained only for a limited number of cases. One example of such an analytical solution can be found in textbooks for an infinite isotropic plate subjected to the initial Gaussian shape: ${ }^{6}$ 
TABLE I. Elastic and geometrical parameters of plates and impactors.

\begin{tabular}{|c|c|c|c|c|}
\hline $\begin{array}{l}\text { Aluminum plates } \\
\rho=2660 \mathrm{~kg} \mathrm{~m}^{-3} \\
\text { plate } a_{1}: \\
\text { plate } a_{3}:\end{array}$ & $\begin{array}{l}D_{1}=6160 \mathrm{MPa} \\
l_{x}=304 \mathrm{~mm} \\
l_{x}=419.5 \mathrm{~mm}\end{array}$ & $\begin{array}{l}D_{4}=8600 \mathrm{MPa} \\
l_{y}=192 \mathrm{~mm} \\
l_{y}=400 \mathrm{~mm}\end{array}$ & $\begin{array}{l}h=2 \mathrm{~mm} \\
h=4 \mathrm{~mm}\end{array}$ & $\begin{array}{l}f_{c}=6 \mathrm{kHz} \\
f_{c}=3 \mathrm{kHz}\end{array}$ \\
\hline $\begin{array}{l}\text { Glass plate } \\
\rho=2550 \mathrm{~kg} \mathrm{~m}^{-3} \\
\text { plate } v_{1}:\end{array}$ & $\begin{array}{l}D_{1}=6700 \mathrm{MPa} \\
l_{x}=229.5 \mathrm{~mm}\end{array}$ & $\begin{array}{l}D_{4}=10270 \mathrm{MPa} \\
l_{y}=220.5 \mathrm{~mm}\end{array}$ & $h=2 \mathrm{~mm}$ & $f_{c}=5.8 \mathrm{kHz}$ \\
\hline $\begin{array}{l}\text { Carbon plate } \\
\rho=1540 \mathrm{~kg} \mathrm{~m}^{-3} \\
\text { plate } c_{1}:\end{array}$ & $\begin{array}{l}D_{1}=8437 \mathrm{MPa} \\
l_{x}=399 \mathrm{~mm}\end{array}$ & $\begin{array}{l}D_{2}=463 \mathrm{MPa} \\
l_{y}=200 \mathrm{~mm}\end{array}$ & $\begin{array}{l}D_{3}=852 \mathrm{MPa} \\
h=2.2 \mathrm{~mm}\end{array}$ & $\begin{array}{l}D_{4}=2267 \mathrm{MPa} \\
\text { critical zone }=[3.6,11.3] \mathrm{kHz}\end{array}$ \\
\hline $\begin{array}{l}\text { Carbon-Epoxy plate } \\
\rho=1581 \mathrm{~kg} \mathrm{~m}^{-3} \\
\text { plate } m_{1}:\end{array}$ & $\begin{array}{l}D_{1}=6912 \mathrm{MPa} \\
l_{x}=70.7 \mathrm{~mm}\end{array}$ & $\begin{array}{l}D_{2}=51.5 \mathrm{MPa} \\
l_{y}=70.7 \mathrm{~mm}\end{array}$ & $\begin{array}{l}D_{3}=4750 \mathrm{MPa} \\
h=2 \mathrm{~mm}\end{array}$ & $D_{4}=1015 \mathrm{MPa}$ \\
\hline $\begin{array}{l}\text { Wooden plate } \\
\rho=388 \mathrm{~kg} \mathrm{~m}^{-3} \\
\text { plate } b_{1}:\end{array}$ & $\begin{array}{l}D_{1}=1013 \mathrm{MPa} \\
l_{x}=515 \mathrm{~mm}\end{array}$ & $\begin{array}{l}D_{2}=27.5 \mathrm{MPa} \\
l_{y}=412.5 \mathrm{~mm}\end{array}$ & $\begin{array}{l}D_{3}=53.7 \mathrm{MPa} \\
h=4.8 \mathrm{~mm}\end{array}$ & $\begin{array}{l}D_{4}=221 \mathrm{MPa} \\
\text { critical zone }=[2.4,10.3] \mathrm{kHz}\end{array}$ \\
\hline $\begin{array}{l}\text { Titanium impactor } \\
\text { radius } R=10 \mathrm{~mm} \\
k_{H}=4.0 \times 10^{-6} \mathrm{~m} \mathrm{~N}^{-2 / 3}\end{array}$ & $m_{i}=1.12 \mathrm{~kg}$ & & & \\
\hline $\begin{array}{l}\text { Rubber impactor } \\
\text { radius } R=10 \mathrm{~mm} \\
k_{H}=9.0 \times 10^{-6} \mathrm{~m} \mathrm{~N}^{-2 / 3}\end{array}$ & $m_{i}=23.6 \mathrm{~g}$ & & & \\
\hline
\end{tabular}

TABLE II. Damping parameters.

Thermoelastic damping (aluminum)

$\begin{array}{ll}\widetilde{D}_{1}(s)=D_{1}\left(1+\frac{s R_{1}}{s+\left(c_{1} / h_{2}\right)}\right), & \widetilde{D}_{4}(s)=D_{4} \\ R_{1}=8.45 \times 10^{-3} & c_{1}=8.0 \times 10^{-4} \mathrm{rad} \mathrm{m}^{2} \mathrm{~s}^{-1}\end{array}$

Viscoelastic damping (glass)

$\widetilde{D}_{i}(s)=D_{i}\left(1+\frac{s R_{1}}{s+s_{1}}+\frac{s R_{2}}{s+s_{2}}\right), \quad i=[1,4]$

$R_{1}=1.63 \times 10^{-3} \quad s_{1}=5180 \mathrm{rad} \mathrm{s}^{-1} \quad R_{2}=1.962 \times 10^{-3} \quad s_{2}=55100 \mathrm{rad} \mathrm{s}^{-1}$

Viscoelastic damping (carbon)

$\begin{array}{llll}\widetilde{D}_{i}(s)=D_{i}\left(1+\frac{s R_{i 1}}{s+s_{i 1}}+\frac{s R_{i 2}}{s+s_{i 2}}\right), & i=[1,3,4] & \widetilde{D}_{2}(s)=D_{2} & \\ R_{11}=1.32 \times 10^{-3} & s_{11}=10.1 \times 10^{3} \mathrm{rad} \mathrm{s}^{-1} & R_{12}=5.0 \times 10^{-3} & \\ R_{31}=8.8 \times 10^{-3} & s_{31}=2.5 \times 10^{3} \mathrm{rad} \mathrm{s}^{-1} & R_{32}=44.0 \times 10^{-3} & s_{12}=94.0 \times 10^{3} \mathrm{rad} \mathrm{s}^{-1} \\ R_{41}=10.4 \times 10^{-3} & s_{41}=2.27 \times 10^{3} \mathrm{rad} \mathrm{s}^{-1} & R_{42}=14.4 \times 10^{-3} & s_{42}=40.0 \times 10^{3} \mathrm{rad} \mathrm{s}^{-1}\end{array}$

Viscoelastic damping (wood)

$\begin{array}{llll}\widetilde{D}_{i}(s)=D_{i}\left(1+\frac{s R_{i 1}}{s+s_{i 1}}+\frac{s R_{i 2}}{s+s_{i 2}}\right), & i=[1,3,4] & \widetilde{D}_{2}(s)=D_{2} & \\ R_{11}=8.18 \times 10^{-3} & s_{11}=3.2 \times 10^{3} \mathrm{rad} \mathrm{s}^{-1} & R_{12}=10.010^{-3} & \\ R_{31}=16.7 \times 10^{-3} & s_{31}=1.1 \times 10^{3} \mathrm{rad} \mathrm{s}^{-1} & R_{32}=70.0 \times 10^{-3} & s_{12}=50.2 \times 10^{3} \mathrm{rad} \mathrm{s}^{-1} \\ R_{41}=15.2 \times 10^{-3} & s_{41}=1.75 \times 10^{3} \mathrm{rad} \mathrm{s}^{-1} & R_{42}=35.0 \times 10^{-3} & s_{42}=50.2 \times 10^{3} \mathrm{rad} \mathrm{s}^{-1}\end{array}$

Radiation damping (isotropic plates)

\begin{tabular}{|c|c|c|}
\hline$\widetilde{D}_{i}(s)=D_{i}\left(1+\frac{2 \rho_{a} c}{\rho h \omega_{c}} \frac{\Sigma_{1}^{3} b_{m}\left(s / \omega_{c}\right)^{m}}{\Sigma_{0}^{3} a_{n}\left(s / \omega_{c}\right)^{n}}\right)$ & $i=[1,4]$ & $\omega_{c}=c^{2} \sqrt{\frac{\rho}{h^{2} D_{1}}}$ \\
\hline$\rho_{a}=1.2 \mathrm{~kg} \mathrm{~m}^{-3}$ & $c=344 \mathrm{~m} \mathrm{~s}^{-1}$ & \\
\hline $\begin{array}{l}a_{0}=1.1669 \\
b_{1}=0.0620\end{array}$ & $\begin{array}{l}a_{1}=1.6574 \\
b_{2}=0.5950\end{array}$ & $\begin{array}{l}a_{2}=1.5528 \\
b_{3}=1.0272\end{array}$ \\
\hline
\end{tabular}

Fluid damping $R_{f}$ in $\mathrm{s}^{-1}$ aluminum $R_{f}=0.032 \quad$ glass $R_{f}=0.88 \quad$ carbon $R_{f}=0.8 \quad$ wood $R_{f}=2.4$ 
TABLE III. General simulation parameters.

Typical time and spatial steps

$f_{e}=1 / \Delta t=192 \mathrm{kHz} \quad \Delta x=\Delta y=9.5 \mathrm{~mm}$

Impact position

$x_{o}=l_{x} / 2 \quad y_{o}=l_{y} / 2$

Listening point

$x=l_{x} / 2$

$y=l_{y} / 2$

$z=24 \mathrm{~cm}$

$$
W(0, r)=W_{0} e^{-r^{2} / a^{2}}
$$

In this case, the displacement at time $t$ is given by:

$$
\begin{aligned}
W(t, r)= & \frac{W_{0}}{1+\tau^{2}} e^{-r^{2} / a^{2}\left(1+\tau^{2}\right)} \\
& \times\left[\cos \frac{r^{2} \tau}{a^{2}\left(1+\tau^{2}\right)}+\tau \sin \frac{r^{2} \tau}{a^{2}\left(1+\tau^{2}\right)}\right],
\end{aligned}
$$

where

$$
\tau=\frac{4 t}{a^{2}} \sqrt{h^{2} D_{1}} \rho .
$$

Figure 3(a) shows the shape of the plate at successive instants of time (between 0 and $0.25 \mathrm{~ms}$ ) for an aluminum plate with thickness $h=2 \mathrm{~mm}$, and for an initial Gaussian shape of width $a=2.5 \mathrm{~cm}$. The other dimensions of the plate are sufficiently large $(1 \mathrm{~m} \times 1 \mathrm{~m})$ so that no reflexions from the boundaries are observed during the selected time scale. No differences can be easily seen between the exact solution [top of Fig. 3(a)] and the simulated solution obtained with

(a)

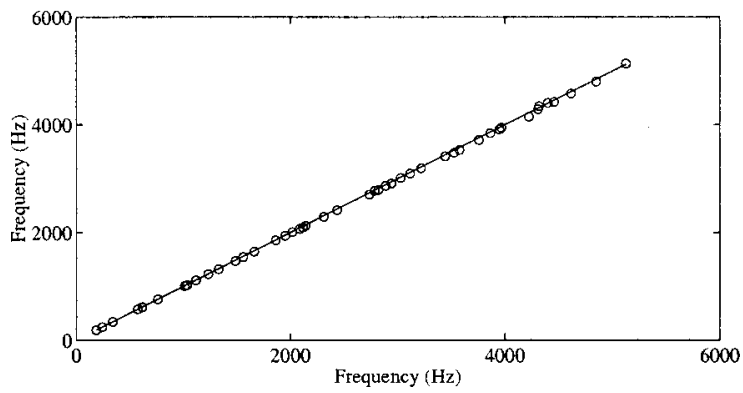

(b)

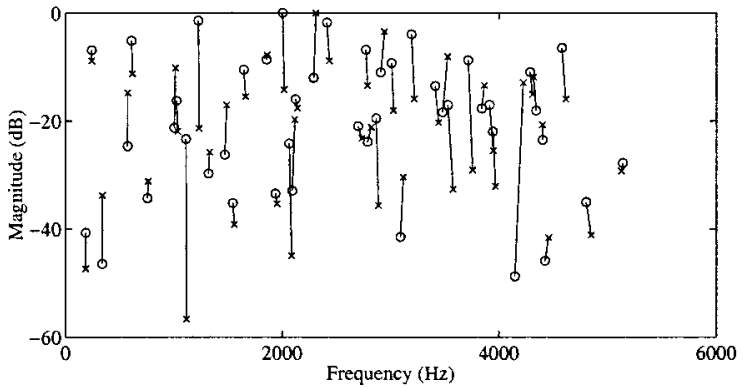

FIG. 6. Aluminum plate. FFT analysis of the sound pressure during the first $20 \mathrm{~ms}$. (a) Simulated vs measured frequencies in the range $0-5 \mathrm{kHz}$ (" $\bigcirc$ "). The solid line indicates perfect matching between the two frequency sets (slope equal to 1). (b) Comparison between simulated (" $X$ ") and measured ("O") magnitudes of the main FFT peaks. (a)

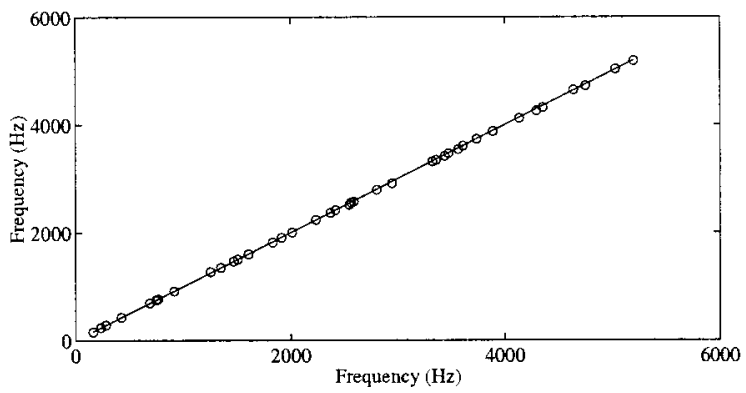

(b)

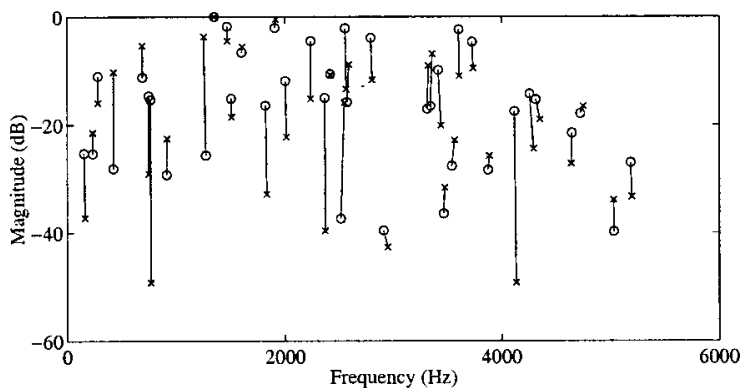

FIG. 7. Glass plate. FFT analysis of the sound pressure during the first 20 ms. (a) Simulated vs measured frequencies in the range $0-5 \mathrm{kHz}$ (" $\bigcirc$ ") The solid line indicates perfect matching between the two frequency sets (slope equal to 1). (b) Comparison between simulated (" $\times$ ") and measured (" $\bigcirc$ ") magnitudes of the main FFT peaks.

(a)

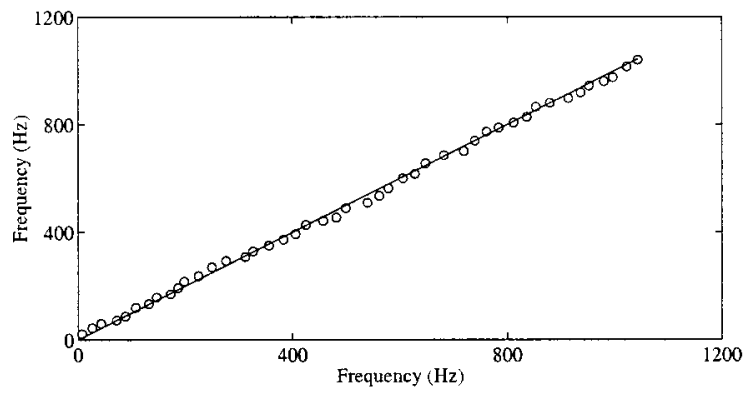

(b)

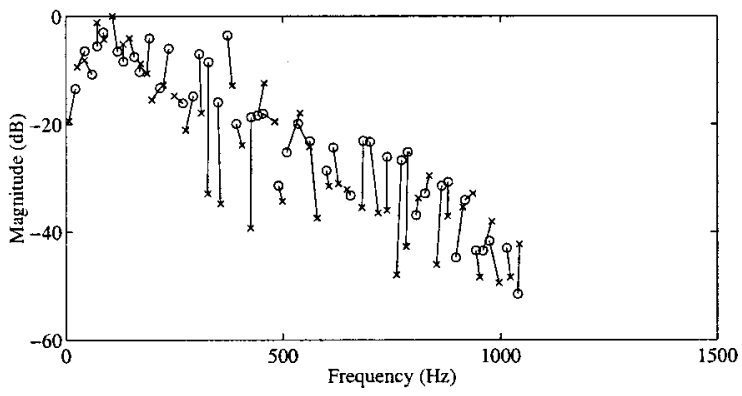

FIG. 8. Carbon plate. FFT analysis of the sound pressure during the first 20 ms. (a) Simulated vs measured frequencies in the range $0-1.5 \mathrm{kHz}$ (" $\bigcirc$ ") The solid line indicates perfect matching between the two frequency sets (slope equal to 1). (b) Comparison between simulated (" $\times$ ") and measured (" $\bigcirc$ ") magnitudes of the main FFT peaks. 
(a)

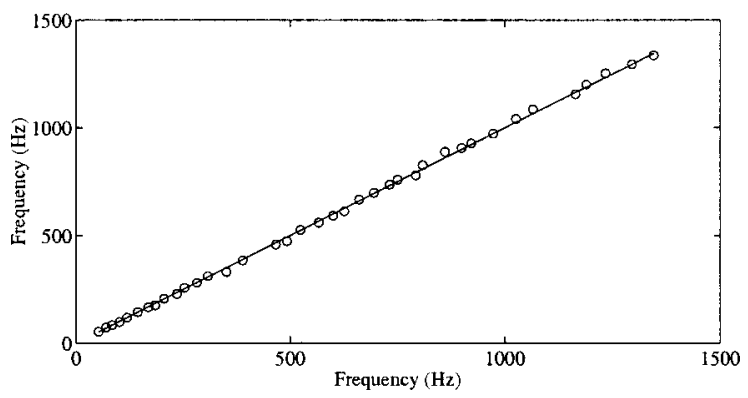

(b)

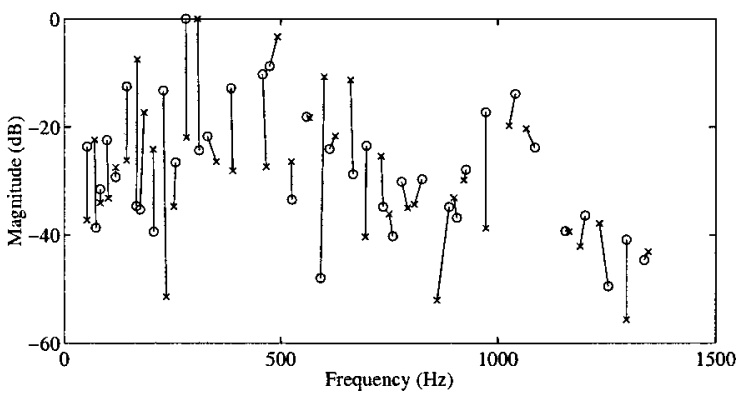

FIG. 9. Wooden plate. FFT analysis of the sound pressure during the first 20 ms. (a) Simulated vs measured frequencies in the range $0-1.5 \mathrm{kHz}$ (" $\bigcirc$ ") The solid line indicates perfect matching between the two frequency sets (slope equal to 1). (b) Comparison between simulated (" $X$ ") and measured (" $O$ ") magnitudes of the main FFT peaks.

our finite-difference scheme [bottom of Fig. 3(a)]. Therefore, in order to better show the discrepancies between these two solutions, Fig. 3(b) shows the relative error between the two waveforms as a function of time for four different sampling frequencies. For a sampling frequency equal to $200 \mathrm{kHz}$, for example, it can be seen that the relative error remains always smaller than $5 \%$ and tends rapidly to less than $1 \%$.

\section{B. Boundary conditions}

Figure 4 illustrates the ability of the numerical method to calculate the flexural vibrations of the plate under various boundary conditions: simply supported [Fig. 4(a)], clamped [Fig. 4(b)] and free plate [Fig. 4(c)]. This figure shows, in addition, that the scheme is able to simulate L-shaped plates, provided that the sides are parallel to the axes. Thus, the model is not restricted to simple rectangular plates.

\section{Impact simulation: Comparison with another method}

In her thesis, McMillan tackles similar problems of impacted plates, using a method based on Green functions of the vibrating structure at the impact point. ${ }^{9}$ Figure 5 shows the simulated forces obtained with the two methods, using the same geometric and elastic parameters, in the case of a Carbon-Epoxy plate struck by a Titanium impactor (see Table I). The force histories obtained with the present finitedifference method are on the left-hand side of the figure, whereas the force histories obtained with the McMillan method are on the right-hand side. Figure 5(a) corresponds to an impact with initial velocity $1 \mathrm{~m} / \mathrm{s}$, whereas Fig. 5(b) cor- responds to an impact velocity of nearly $0.6 \mathrm{~m} / \mathrm{s}$. It can be seen that the two methods yield identical waveforms. The ripples in the force pulses are due to the fact that the inverse of the lowest eigenfrequency of the plate here is about 20 times lower than the interaction time, a consequence of the particular selected set of parameters.

\section{Comparison between measured and simulated pressure}

The simulated sounds are now compared with measured sounds. Due to the large number of excited modes in each case, no quantitative information can be easily derived from the visual comparison between measured and simulated waveforms. In this respect, comparisons in the spectral domain are more informative. All spectra are calculated by FFT (fast Fourier transform) on the first $20 \mathrm{~ms}$ on the sound and normalized (in $\mathrm{dB}$ ) with respect to the magnitude of the highest peak. In order to allow quantitative comparison between measured and simulated spectra, only the most significant peaks are displayed in the figures. These data are extracted from the FFT analysis by means of a simple peak detection algorithm. The values of the parameters used for the simulations can be found in Tables I-III.

Figure 6 shows the results obtained for an aluminum plate excited with a xylophone rubber mallet with initial velocity $1.45 \mathrm{~m} / \mathrm{s}$. Figure 6(a) shows that the number and frequencies of the spectral peaks, between 0 and $5 \mathrm{kHz}$, are very well reproduced. The spectral envelopes of both real and simulated sounds are comparable. The displayed spectral domain is limited here to $5 \mathrm{kHz}$ for reasons of clarity, and also because the magnitude of the peaks above $5 \mathrm{kHz}$ are relatively small (less than $-40 \mathrm{~dB}$ below the maximum), a consequence of both excitation spectrum and damping. The damping here is mainly due to thermoelastic and radiation losses. ${ }^{1}$

Figure 7 shows the results obtained for a glass plate. Here again, the measured and simulated frequencies and magnitudes of the spectral peaks look very similar, which is confirmed by listening to the corresponding sounds. The losses in this material are mainly due to viscoelasticity and radiation. ${ }^{1}$

A first example of impact against an orthotropic plate is given in Fig. 8 which compares measurements with simulations in the case of a plate made of carbon fibers. It can be seen on the spectra that the high frequencies are rapidly damped: the magnitude of the peaks are less than $-40 \mathrm{~dB}$ below the maximum for frequencies above $1.2 \mathrm{kHz}$. Here, the impact produces a duller sound than with aluminum and glass. The damping is almost entirely due to viscoelastic losses in the material, and the radiation losses can be neglected. ${ }^{1}$ Similar conclusions can be drawn from Fig. 9 which shows the results obtained in the case of wood (Spruce). Here, the damping of the vibrations in the plate is mainly due to viscoelastic losses. However, due to the dimensions of the plate, the radiation losses cannot be totally neglected. The discrepancies between measurements and simulations for some of the peaks are probably due to the approximations made in the radiation model. 
(a) Aluminum

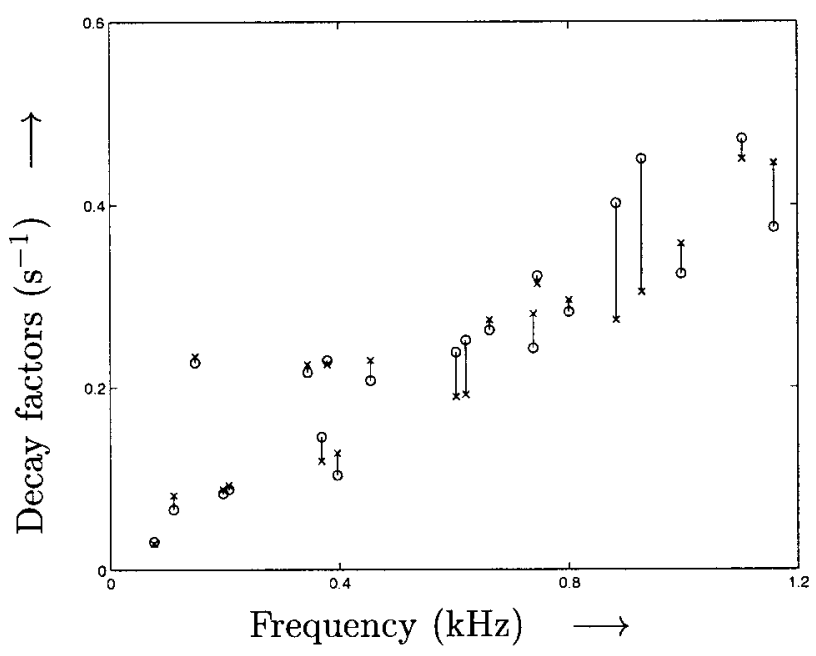

(c) Carbon fibers

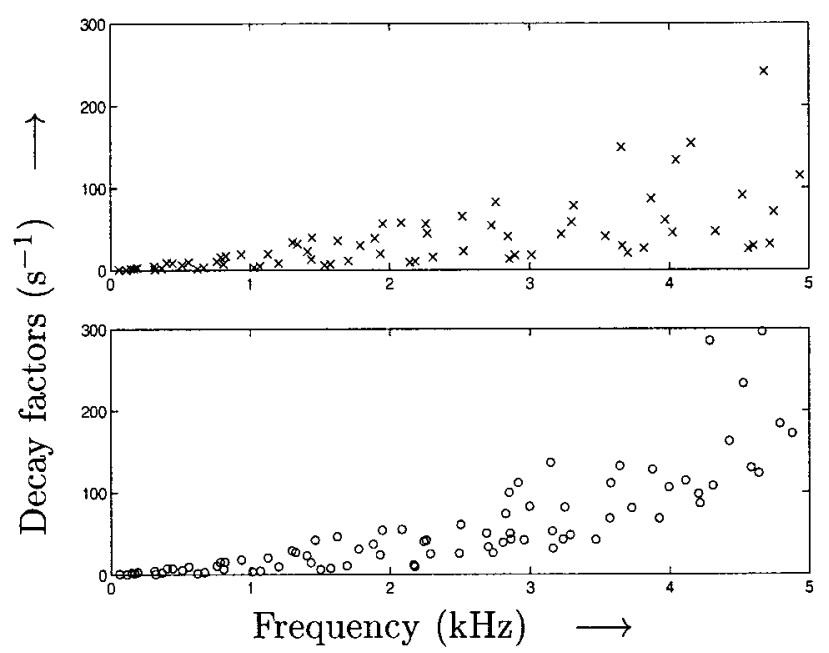

(b) Aluminum

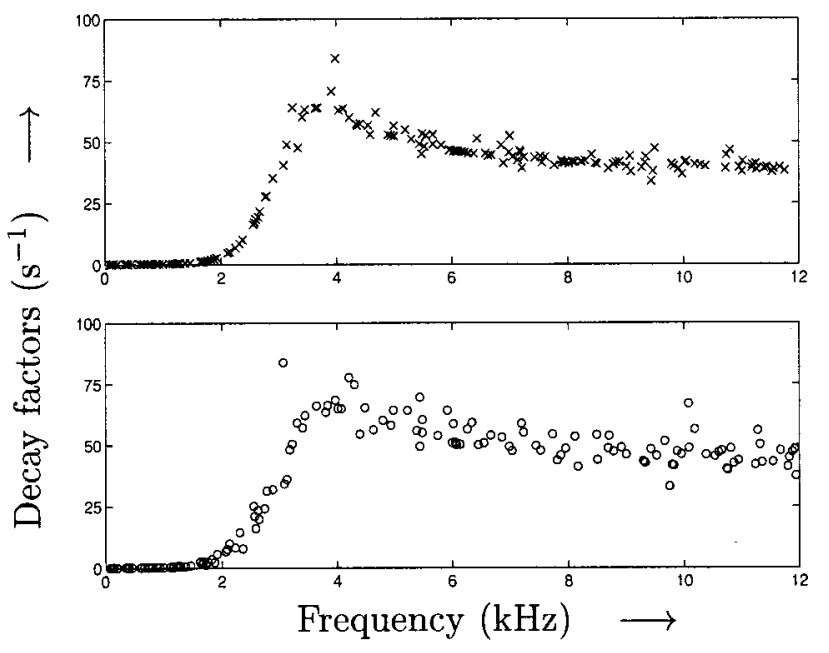

(d) Wood

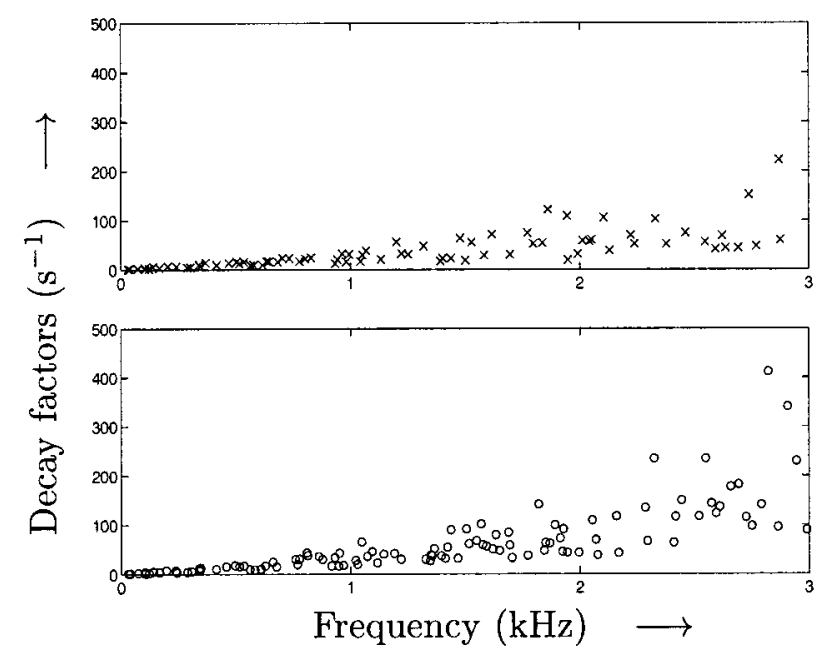

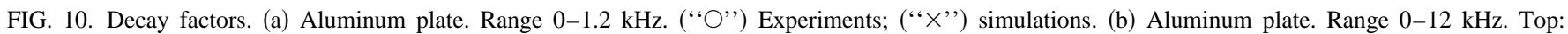
simulations; bottom: measurements. (c) Carbon plate. Range 0-5 kHz. Top: simulations; bottom: measurements. (d) Wooden plate. Range 0-3 kHz. Top: simulations; bottom: measurements.

In a previous paper, measured decay factors were compared to the values predicted by the continuous plate model solved in the frequency domain. ${ }^{1}$ To completely assess the validity of the method, Fig. 10 shows, in addition, the comparison between measured and simulated decay factors, where the simulated damping factors are now obtained from a frequency analysis of the simulated waveforms. The analysis method used here is the Matrix Pencil method. ${ }^{17}$ Figure 10(a) shows that the thermoelastic model of losses accounts for the apparent erratic distribution of the decay factors in the low-frequency range for metallic plates. This distribution essentially follows from the fact that the decay time of each eigenfrequency depends on its modal shape. In this frequency range (below $2 \mathrm{kHz}$, for the plate shown on this figure) the radiation damping is negligible. The low values of the decay factors below the critical frequency play a major role in duration and tone quality of the sound generated by the free vibrations of the plate. Figure 10(b) shows the relevance of the asymptotic radiation model which accounts for the losses above the critical frequency of the plate. For this plate (aluminum $a_{3}$, see parameters in Tables I and II), the average decay factor above $4 \mathrm{kHz}$ is about 100 times the average decay factor below $1 \mathrm{kHz}$.

Figures 10(c) and (d) show the relevance of the viscoelastic model for orthotropic plates. It can be seen that the viscoelastic losses fully account for the measured decay factors, except for the wooden plate above $2 \mathrm{kHz}$ where the radiation losses are not negligible. Here again, the model is able to reproduce the apparent erratic distribution of decay factors with frequency. This distribution is not due to error in the measurements (a commonly erroneous conclusion) but rather to the fact that there is a specific damping model for each complex rigidity (see Table II). Notice further that 13 damping parameters only are needed here for reproducing adequately the temporal evolution of more than 40 modes. This is an interesting feature of the method in terms of data reduction. 


\section{CONCLUSION}

A time-domain model for the simulation of flexural vibrations and sounds radiated by damped isotropic and orthotropic plates has been presented. In order to demonstrate the particular interest of the method for the synthesis of transients, the study was made for plates excited by an impact.

The key point of the model is that the losses in the plates are modeled in the time domain with the help of a general differential operator applied to the rigidities of the material. This formulation gives great flexibility for modeling the damping of plates. As shown in a companion paper, this formulation allows the modeling of the three main mechanisms of damping in plates: viscoelasticity, thermoelasticity, and radiation. ${ }^{1}$ Therefore, it makes it possible to reproduce adequately the complicated frequency dependence of the decay times observed in a large variety of materials with only a small number of damping parameters.

The sounds synthesized with this model allow a clear recognition of the materials without ambiguity. This accurate reproduction would not be possible with a simple description of the damping, as with a single loss factor, for example. All measured and simulated sounds presented in this paper can be heard at the following Web address: http:// wwwy.ensta.fr/ chaigne/plaque/

compar_exp_sim.html.

The equations are solved numerically by means of a finite-difference scheme of second order in time and fourth order in space (2-4 scheme). The presence of complex rigidities makes it necessary to investigate thoroughly the stability of the scheme, which contributes to filling a gap in the literature. It has been shown that a weak stability condition can be obtained from the study of the amplification matrix, following the method by Richtmyer and Morton. ${ }^{14}$

The accuracy of the method is studied in terms of numerical dispersion. A comparison with another scheme of lower order in space (2-2 scheme) shows that, for a given accuracy, the required computing time with the selected 2-4 scheme is about 4 times lower than with the 2-2 scheme.

In order to show the validity of the method, a comparison is made first between an exact analytical solution and the numerical result obtained for an infinite undamped isotropic plate. The impact model is further validated by comparing the calculated force pulses imparted to the plate with the force pulses obtained with another method. ${ }^{9}$ Finally, simulated sounds are systematically compared to measurements for four different materials. These comparisons show a particularly good agreement in terms of frequencies and damping factors despite the relatively low number of damping parameters inserted in the model.

The present model is limited to rectangular plates and to plates with rectangular corners (see Fig. 4), for which the use of finite differences is particularly well-suited. Extension of this model to more complicated shapes makes it necessary to use other numerical methods, such as finite elements based on a variational formulation of the problem. ${ }^{18}$

\section{APPENDIX A: FINITE-DIFFERENCE OPERATORS}

The discrete operators given below are obtained by linear combinations of Taylor expansions of the field variable $v(x, y, t)$. In this paper, $\left(D_{t^{p}}^{(i)} v\right)_{l, m}^{n}$ denotes the approximation of the $p$ th time derivative of $v$ at point $(l \Delta x, m \Delta y, n \Delta t)$, where the subscripts $(i)$ is the order of the truncation error in $\Delta t$.

\section{First order decentered operators}

The following operators are used to approximate the time-derivatives in the damping terms.

$$
\begin{aligned}
&\left(D_{t}^{(1)} v\right)^{n}=\frac{1}{\Delta t}\left(v^{n}-v^{n-1}\right)=\left(v_{l, m}^{n}\right)_{, t}+O(\Delta t) \\
&\left(D_{t t}^{(1)} v\right)^{n}=\left(D_{t}^{(1)} o D_{t}^{(1)} v\right)^{n} \\
&=\frac{1}{\Delta t^{2}}\left(v^{n}-2 v^{n-1}+v_{n-2}\right)=\left(v_{l, m}^{n}\right)_{, t t}+O(\Delta t) \\
&\left(D_{t^{3}}^{(1)} v\right)^{n}=\left(D_{t}^{(1)} o D_{t}^{(1)} o D_{t}^{(1)} v\right)^{n} \\
&=\frac{1}{\Delta t^{3}}\left(v^{n}-3 v^{n-1}+3 v^{n-2}-v^{n-3}\right) \\
&=\left(v_{l, m}^{n}\right)_{, t t t}+O(\Delta t) . \\
& \vdots
\end{aligned}
$$

\section{Second order centered operators}

$$
\begin{aligned}
\left(D_{t t}^{(2)} v\right)_{l, m}^{n}= & \frac{1}{\Delta t^{2}}\left(v_{l, m}^{n+1}+v_{l, m}^{n-1}-2 v_{l, m}^{n}\right) \\
= & \left(v_{l, m}^{n}\right)_{, t t}+O\left(\Delta t^{2}\right) \\
\left(D_{x x}^{(2)} v\right)_{l, m}^{n}= & \frac{1}{\Delta x^{2}}\left(v_{l+1, m}^{n}+v_{l-1, m}^{n}-2 v_{l, m}^{n}\right) \\
= & \left(v_{l, m}^{n}\right)_{, x x}+O\left(\Delta x^{2}\right), \\
\left(D_{y y}^{(2)} v\right)_{l, m}^{n}= & \frac{1}{\Delta y^{2}}\left(v_{l, m+1}^{n}+v_{l, m-1}^{n}-2 v_{l, m}^{n}\right) \\
= & \left(v_{l, m}^{n}\right)_{, y y}+O\left(\Delta y^{2}\right), \\
\left(D_{x y}^{(2)} v\right)_{l+1 / 2, m+1 / 2}^{n}= & \frac{1}{\Delta x \Delta y}\left(v_{l+1, m+1}^{n}+v_{l, m}^{n}\right. \\
& \left.-v_{l+1, m}^{n}-v_{l, m+1}^{n}\right) \\
= & \left(v_{l+1 / 2, m+1 / 2}^{n}\right)_{, x y}+O\left(\Delta x^{2}\right)+O\left(\Delta y^{2}\right),
\end{aligned}
$$

$$
\begin{aligned}
\left(\widetilde{D}_{x y}^{(2)} v\right)_{l, m}^{n}= & \frac{1}{\Delta x \Delta y}\left(v_{l+1 / 2, m+1 / 2}^{n}+v_{l-1 / 2, m-1 / 2}^{n}\right. \\
& \left.-v_{l+1 / 2, m-1 / 2}^{n}-v_{l-1 / 2, m+1 / 2}^{n}\right) \\
= & \left(v_{l, m}^{n}\right)_{, x y}+O\left(\Delta x^{2}\right)+O\left(\Delta y^{2}\right) .
\end{aligned}
$$




$$
\begin{aligned}
\left(D_{x x}^{(4)} v\right)_{l, m}^{n}= & \frac{1}{12 \Delta x^{2}}\left[-\left(v_{l+2, m}^{n}+v_{l-2, m}^{n}\right)\right. \\
& \left.+16\left(v_{l+1, m}^{n}+v_{l-1, m}^{n}\right)-30 v_{l, m}^{n}\right] \\
= & \left(v_{l, m}^{n}\right)_{, x x}+O\left(\Delta x^{4}\right),
\end{aligned}
$$

$$
\begin{aligned}
\left(D_{y y}^{(4)} v\right)_{l, m}^{n}= & \frac{1}{12 \Delta y^{2}}\left[-\left(v_{l, m+2}^{n}+v_{l, m-2}^{n}\right)\right. \\
& \left.+16\left(v_{l, m+1}^{n}+v_{l, m-1}^{n}\right)-30 v_{l, m}^{n}\right] \\
= & \left(v_{l, m}^{n}\right)_{, y y}+O\left(\Delta y^{4}\right)
\end{aligned}
$$

$$
\begin{aligned}
\left(D_{x y}^{(4)} v\right)_{l+1 / 2, m+1 / 2}^{n}= & \frac{1}{24^{2} \Delta x \Delta y}\left[\left(v_{l-1, m-1}^{n}+v_{l+2, m+2}^{n}-v_{l-1, m+2}^{n}-v_{l+1, m-1}^{n}\right)+27\left(v_{l-1, m+1}^{n}+v_{l, m+2}^{n}+v_{l+1, m-1}^{n}+v_{l+2, m}^{n}\right)\right. \\
& \left.-27\left(v_{l-1, m}^{n}+v_{l, m-1}^{n}+v_{l+1, m+2}^{n}+v_{l+2, m+1}^{n}\right)+27^{2}\left(v_{l, m}^{n}+v_{l+1, m+1}^{n}-v_{l+1, m}^{n}-v_{l, m+1}^{n}\right)\right] \\
= & \left(v_{l+1 / 2, m+1 / 2}^{n}\right)_{, x y}+O\left(\Delta x^{4}\right)+O\left(\Delta y^{4}\right)
\end{aligned}
$$

$$
\begin{aligned}
\left(\widetilde{D}_{x y}^{(4)} v\right)_{l, m}^{n}= & \frac{1}{24^{2} \Delta x \Delta y}\left[\left(v_{l-3 / 2, m-3 / 2}^{n}+v_{l+3 / 2, m+3 / 2}^{n}-v_{l+3 / 2, m-3 / 2}^{n}-v_{l-3 / 2, m+3 / 2}^{n}\right)+27\left(v_{l-3 / 2, m+1 / 2}^{n}+v_{l-1 / 2, m+3 / 2}^{n}\right.\right. \\
& \left.+v_{l+1 / 2, m-3 / 2}^{n}+v_{l+3 / 2, m-1 / 2}^{n}\right)-27\left(v_{l-3 / 2, m-1 / 2}^{n}+v_{l-1 / 2, m-3 / 2}^{n}+v_{l+1 / 2, m+3 / 2}^{n}+v_{l+3 / 2, m+1 / 2}^{n}\right) \\
& \left.+27^{2}\left(v_{l-1 / 2, m-1 / 2}^{n}+v_{l+1 / 2, m+1 / 2}^{n}-v_{l+1 / 2, m-1 / 2}^{n}-v_{l-1 / 2, m+1 / 2}^{n}\right)\right]=\left(v_{l, m}^{n}\right)_{, x y}+O\left(\Delta x^{4}\right)+O\left(\Delta y^{4}\right) .
\end{aligned}
$$

\section{APPENDIX B: STABILITY}

\section{1D problem}

By introducing the spatial Fourier transforms of both the displacement $w_{n} \exp (j k l \Delta x)$ and bending moment $\left(m_{x}\right)_{n} \exp (j k l \Delta x)$, Eq. (19) can be rewritten in the following matrix form:

$$
\mathbf{v}_{\mathbf{n}+1}=\widetilde{\mathbf{G}}(\Delta \mathbf{t}, \mathbf{k}) \mathbf{v}_{\mathbf{n}}
$$

with

$$
\mathbf{v}_{\mathbf{n}+\mathbf{1}}=\left[w_{n+1}, w_{n}, \cdots, w_{n-N+1},\left(n_{x}\right)_{n}, \cdots,\left(n_{x}\right)_{n-N+1}\right]^{T},
$$

where $\left(n_{x}\right)_{n}=\Delta x^{2}\left(m_{x}\right)_{n}$. The amplification matrix $\widetilde{G}(\Delta t, k)$ is given by:

$$
\left(\begin{array}{cccccccccc}
A & -B C \chi_{2} & \cdots & \cdots & -B C \chi_{N} & C \psi_{1} & \cdots & \cdots & \cdots & C \psi_{N} \\
1 & 0 & \cdots & \cdots & 0 & 0 & \cdots & \cdots & \cdots & 0 \\
0 & 1 & 0 & \cdots & 0 & \vdots & & & & \vdots \\
\vdots & & \ddots & & \vdots & \vdots & & & & \vdots \\
0 & \cdots & 0 & 1 & 0 & 0 & \cdots & \cdots & \cdots & 0 \\
B \chi_{0} & \cdots & \cdots & \cdots & B \chi_{N} & -\psi_{1} & \cdots & \cdots & \cdots & -\psi_{N} \\
0 & \cdots & \cdots & \cdots & 0 & 1 & 0 & \cdots & \cdots & 0 \\
\vdots & & & & \vdots & 0 & 1 & 0 & \cdots & 0 \\
\vdots & & & & \vdots & \vdots & & \ddots & & \vdots \\
0 & \cdots & \cdots & \cdots & 0 & 0 & \cdots & 0 & 1 & 0
\end{array}\right)
$$

with

$$
P=2-R_{f} \Delta t, \quad B=4 I E X^{2}, \quad C=\frac{4 X^{2} \Delta t^{2}}{\rho S \Delta x^{4}}, \quad F=R_{f} \Delta t-1, \quad X=\sin \frac{k \Delta x}{2}, \quad A=\left(P-B C \chi_{0}\right)\left(F-B C \chi_{1}\right) .
$$


The discrete system in Eq. (B1) is called weakly stable if there exists a real constant $K(\tau, T)$ such that: ${ }^{14}$

$$
\left\|\widetilde{\mathbf{G}}(\Delta \mathbf{t}, \mathbf{k})^{\mathbf{n}}\right\| \leqslant K(\tau, T), \text { for all }\left\{\begin{array}{l}
0<\Delta t<\tau \\
0 \leqslant n \Delta t \leqslant T, \\
k \text { real }
\end{array}\right.
$$

where $\|\widetilde{G}(\Delta t, k)\|$ denotes the spectral norm of the matrix $\widetilde{G}(\Delta t, k)$. A condition for strong stability is obtained by replacing $K(\tau, T)$ by 1 in Eq. (B5). The following criterion has been applied here: ${ }^{14}$

"If $\widetilde{G}(\Delta t, k)$ is uniformly Lipschitz continuous for $\quad \Delta t=0, \quad$ so far as $\widetilde{G}(\Delta t, k)=\widetilde{G}(0, k)$ $+O(\Delta t)$, as $\Delta t \rightarrow 0$, where the constant implied by the expression $O(\Delta t)$ does not depend on $k$, then the scheme is weakly stable if and only if $\widetilde{G}(0, k)$ is stable."

To apply this criterion, the first step consists of studying the dependence of $\widetilde{G}(\Delta t, k)$ on $\Delta t$. This parameter appears in the coefficients $A, C$ and $F$ defined in Eq. (B4) and in the expressions of the coefficients $\chi_{r}$ and $\psi_{r}$ :

$$
\begin{aligned}
& \chi_{r}=(-1)^{r} \frac{p_{N}}{q_{N}}+O(\Delta t) \\
& \psi_{r}=(-1)^{r}+O(\Delta t) .
\end{aligned}
$$

All the coefficients of the amplification matrix can be expressed as $g_{i j}+O(\Delta t)$ and thus $\widetilde{G}$ is uniformly Lipschitz continuous. The determination of a stability condition now consists of the study of $\widetilde{G}(0, k)$. It must be checked whether the spectral radius of this matrix remains smaller than or equal to unity for all $k$ or not. The eigenvalues $\lambda_{i}$ of $\widetilde{G}(0, k)$ are such that the vector:

$$
\left(\begin{array}{c}
\left(n_{x}\right)_{n} \\
w_{n}
\end{array}\right)=\lambda_{i}\left(\begin{array}{c}
\left(n_{x}\right)_{n-1} \\
w_{n-1}
\end{array}\right)
$$

is a solution of the discrete system in Eq. (19) for $\Delta t=0$, given by:

$$
\left(\begin{array}{cc}
\sum_{r=1}^{N}(-1)^{r} \lambda_{i}^{-r} & -B \frac{p_{N}}{q_{N}} \sum_{r=0}^{N}(-1)^{r} \lambda_{i}^{-r} \\
C & \lambda_{i}-2+\lambda_{i}^{-1}
\end{array}\right)\left(\begin{array}{c}
\left(n_{x}\right)_{n} \\
w_{n}
\end{array}\right)=\left(\begin{array}{l}
0 \\
0
\end{array}\right) .
$$

The nontrivial solutions of this system are obtained by zeroing the determinant of the matrix:

$$
\left(\sum_{r=0}^{N}(-1)^{r} \lambda_{i}^{r}\right)\left(\lambda_{i}^{2}+\left(\frac{X^{4}}{\xi_{s}} \frac{I E p_{N}}{\rho S q_{N}}-2\right) \lambda_{i}+1\right)=0
$$

from which the following necessary condition for weak stability is obtained:

$$
\Delta x^{2} \geqslant 2 \Delta t \sqrt{\frac{I E p_{N}}{\rho S q_{N}}} .
$$

\section{2D problem}

A necessary condition for the system Eq. (9) to be stable is now determined in the same way as for the $1 \mathrm{D}$ case. It is assumed that the dissipativity conditions on the coefficients $p_{i N}$ and $q_{N}$ are fulfilled (see Ref. 1). As $\Delta t$ tends to zero, $\chi_{i r}$ and $\psi_{i r}$ can be expanded as follows:

$$
\begin{gathered}
\chi_{i r}=(-1)^{r} \frac{p_{i N}}{q_{N}}+O(\Delta t), \quad 1 \leqslant i \leqslant 4 \\
\psi_{i r}=(-1)^{r}+O(\Delta t), \quad i=[1,4] .
\end{gathered}
$$

As for the 1D case, it can be shown that the amplification matrix is Lipschitz-continuous for $\Delta t=0$. The eigenvalues $\lambda_{i}$ of $\widetilde{G}\left(0, k_{x}, k_{y}\right)$ are now solutions of the following system:

$$
\begin{gathered}
\left(\begin{array}{cccc}
\mathcal{Q}\left(\lambda_{i}\right) & 0 & 0 & -\left(\mathcal{P}_{1}\left(\lambda_{i}\right)-\mathcal{P}_{21}\left(\lambda_{i}\right)\right) \\
0 & \mathcal{Q}\left(\lambda_{i}\right) & 0 & -\left(\mathcal{P}_{3}\left(\lambda_{i}\right)-\mathcal{P}_{23}\left(\lambda_{i}\right)\right) \\
0 & 0 & \mathcal{Q}\left(\lambda_{i}\right) & -\mathcal{P}_{4}\left(\lambda_{i}\right) \\
C_{1} & C_{3} & C_{4} & \mathcal{P}\left(\lambda_{i}\right)
\end{array}\right) \\
\quad\left(\begin{array}{c}
\left(n_{x}\right)_{n} \\
\left(n_{y}\right)_{n} \\
\left(n_{x y}\right)_{n} \\
w_{n}
\end{array}\right)=\left(\begin{array}{l}
0 \\
0 \\
0 \\
0
\end{array}\right),
\end{gathered}
$$

with:

$\mathcal{Q}\left(\lambda_{i}\right)=\sum_{r=0}^{N}(-1)^{r} \lambda_{i}^{-r}, \quad \mathcal{P}_{23}\left(\lambda_{i}\right)=2 h^{3} D_{2} X^{2} \frac{p_{2 N}}{q_{N}} \mathcal{Q}\left(\lambda_{i}\right)$,

$C_{1}=\frac{4 X^{2}}{\rho h \xi_{s}^{2}}, \quad \mathcal{P}\left(\lambda_{i}\right)=\lambda_{i}-2+\lambda_{i}^{-1}$,

$\mathcal{P}_{3}\left(\lambda_{i}\right)=\frac{4 h^{3} D_{3} Y^{2}}{r_{1}^{2}} \frac{p_{3 N}}{q_{N}} \mathcal{Q}\left(\lambda_{i}\right), \quad C_{3}=\frac{4 Y^{2}}{\rho h \xi_{s}^{2} r_{1}^{2}}$,

$\mathcal{P}_{1}\left(\lambda_{i}\right)=4 h^{3} D_{1} X^{2} \frac{p_{1 N}}{q_{N}} \mathcal{Q}\left(\lambda_{i}\right)$,

$\mathcal{P}_{4}\left(\lambda_{i}\right)=\frac{2 h^{3} D_{4} X Y}{r_{1}} \frac{p_{4 N}}{q_{N}} \mathcal{Q}\left(\lambda_{i}\right), \quad C_{4}=\frac{8 X Y}{\rho h \xi_{s}^{2} r_{1}}$,

$\mathcal{P}_{21}\left(\lambda_{i}\right)=\frac{2 h^{3} D_{2} Y^{2}}{r_{1}^{2}} \frac{p_{2 N}}{q_{N}} \mathcal{Q}\left(\lambda_{i}\right), \quad \xi_{s}=\frac{\Delta x^{2}}{\Delta t}, \quad r_{1}=\frac{\Delta y}{\Delta x}$.

As for the 1D case, the determination of the stability condition is obtained by zeroing the determinant of the matrix Eq. (B12) which amounts to studying the roots of the polynomial:

$$
\begin{aligned}
\mathcal{P}(\lambda) & +C_{1}\left(A_{1} \frac{p_{1 N}}{q_{N}}+A_{21} \frac{p_{2 N}}{q_{N}}\right)+C_{3}\left(A_{3} \frac{p_{3 N}}{q_{N}}+A_{23} \frac{p_{2 N}}{q_{N}}\right) \\
+ & C_{4} A_{4} \frac{p_{4 N}}{q_{N}}=0,
\end{aligned}
$$

with

$$
A_{1}=h^{3} D_{1} X, \quad Y=\sin \frac{k \Delta y}{2}, \quad A_{21}=\frac{h^{3} D_{2} Y^{2}}{2 r_{1}^{2}},
$$




$$
A_{3}=\frac{h^{3} D_{3} Y^{2}}{r_{1}^{2}}, \quad A_{23}=\frac{h^{3} D_{2} X^{2}}{2}, \quad A_{4}=\frac{h_{3} D_{4} X Y}{2 r_{1}} .
$$

This yields a stability condition similar to Eq. (17), where the $D_{i}$ are replaced by $D_{i} p_{i N} / q_{N}$.

${ }^{1}$ A. Chaigne and C. Lambourg, "Time-domain simulation of damped impacted plates. Part I. Theory and experiments," J. Acoust. Soc. Am. 109, 1422-1432 (2001).

${ }^{2}$ A. Frendi, L. Maestrello, and A. Bayliss, "Coupling between plate vibration and acoustic radiation," J. Sound Vib. 177, 207-226 (1994).

${ }^{3}$ S. Schedin, C. Lambourg, and A. Chaigne, "Transient sound fields from impacted plates: Comparison between numerical simulations and experiments,"' J. Sound Vib. 221, 471-490 (1999).

${ }^{4}$ V. Doutaut, D. Matignon, and A. Chaigne, "Numerical simulations of xylophones. II. Time-domain modeling of the resonator and of the radiated sound pressure," J. Acoust. Soc. Am. 104, 1633-1647 (1998).

${ }^{5}$ A. W. Leissa, Vibrations of Plates, NASA SP-160 (NASA, Washington, D.C., 1969).

${ }^{6}$ K. F. Graff, Wave Motion in Elastics Solids (Dover, New York, 1991).

${ }^{7}$ A. E. H. Love, A Treatise on the Mathematical Theory of Elasticity (Dover, New York, 1944).

${ }^{8}$ A. Chaigne and V. Doutaut, "Numerical simulations of xylophones. I. Time-domain modeling of the vibrating bars," J. Acoust. Soc. Am. 101, 539-557 (1996).
${ }^{9}$ A. J. McMillan, "A Theoretical Investigation of the Role of Vibration in Elastic Impact," Ph.D. thesis, Staffordshire Polytechnic, 1992.

${ }^{10}$ W. F. Ames, Numerical Methods for Partial Differential Equations, 3rd ed. (Academic, New York, 1992).

${ }^{11}$ S. J. Fenves, N. Perrone, A. R. Robinson, and W. C. Schnobrich, Numerical and Computer Methods in Structural Mechanics (Academic, New York, 1973).

${ }^{12}$ C. J. Moore, An Introduction to the Psychology of Hearing, 2nd ed. (Academic, New York, 1982).

${ }^{13} \mathrm{C}$. Lambourg, "Modèle temporel pour la simulation numérique de plaques vibrantes. Application à la synthèse sonore,' Ph.D. thesis, Université du Maine, 1997.

${ }^{14}$ R. D. Richtmyer and K. W. Morton, Difference Methods for Initial-Value Problems, 2nd ed. (Interscience, New York, 1967).

${ }^{15} \mathrm{G}$. Cohen, Ed., Ecole des Ondes Inria: Méthodes numériques d'ordre élevé pour les ondes en régime transitoire (Collection Didactique, INRIA, 1994).

${ }^{16}$ R. F. S. Hearmon, An Introduction to Applied Anisotropic Elasticity (Oxford University Press, New York, 1961).

${ }^{17}$ J. Laroche, "The use of the matrix pencil method for the spectrum analysis of musical signals," J. Acoust. Soc. Am. 94, 1958-1965 (1993).

${ }^{18}$ L. Rhaouti, A. Chaigne, and P. Joly, "Time-domain modeling and numerical simulation of a kettledrum,' J. Acoust. Soc. Am. 105, 3545-3562 (1999). 